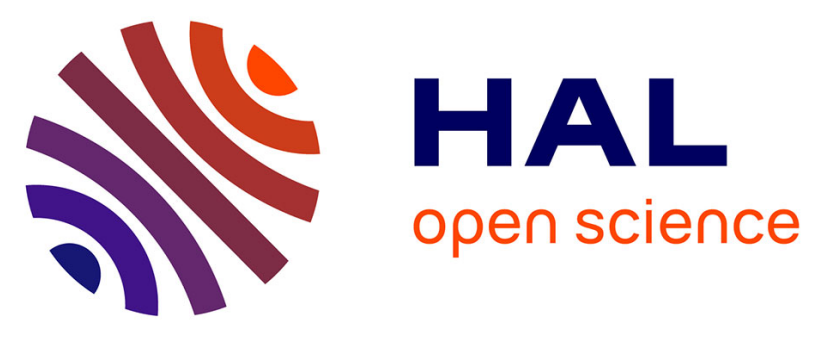

\title{
A new subtype of bone sarcoma defined by BCOR-CCNB3 gene fusion
}

Gaëlle Pierron, Franck Tirode, Carlo Lucchesi, Stéphanie Reynaud, Stelly Ballet, Sarah Cohen-Gogo, Virginie Perrin, Jean-Michel Coindre, Olivier Delattre

\section{To cite this version:}

Gaëlle Pierron, Franck Tirode, Carlo Lucchesi, Stéphanie Reynaud, Stelly Ballet, et al.. A new subtype of bone sarcoma defined by BCOR-CCNB3 gene fusion. Nature Genetics, 2012, 44 (4), pp.461-466. 10.1038/ng.1107 . inserm-02440379

\section{HAL Id: inserm-02440379 https://www.hal.inserm.fr/inserm-02440379}

Submitted on 15 Jan 2020

HAL is a multi-disciplinary open access archive for the deposit and dissemination of scientific research documents, whether they are published or not. The documents may come from teaching and research institutions in France or abroad, or from public or private research centers.
L'archive ouverte pluridisciplinaire HAL, est destinée au dépôt et à la diffusion de documents scientifiques de niveau recherche, publiés ou non, émanant des établissements d'enseignement et de recherche français ou étrangers, des laboratoires publics ou privés. 


\section{A new subtype of bone sarcoma defined by BCOR-CCNB3 gene fusion}

Gaëlle PIERRON ${ }^{1 *}$, Franck TIRODE ${ }^{*}$, Carlo LUCCHESI ${ }^{*}$, Stéphanie REYNAUD ${ }^{1}$, Stelly BALLET ${ }^{1}$, Sarah COHEN-GOGO², Virginie PERRIN², Jean-Michel COINDRE², Olivier DELATTRE $1,2, * *$

* These authors contributed equally to this work

${ }^{1}$ Institut Curie, Unité de Génétique Somatique - Centre Hospitalier, 26 rue d’Ulm 75248 PARIS CEDEX 05

${ }^{2}$ Unité 830 INSERM/Institut Curie - Centre de Recherche, 26 rue d’Ulm 75248 PARIS CEDEX 05

${ }^{3}$ Institut Bergonié, 229 Cours de l'Argonne 33076 BORDEAUX CEDEX

** to whom correspondence should be addressed at:

Unité 830 INSERM/Institut Curie - Centre de Recherche, 26 rue d'Ulm 75248 PARIS CEDEX 05

tel : +33156246679

Fax : +33156246630

email : olivier.delattre@curie.fr 


\begin{abstract}
The identification of subtype-specific translocations has revolutionized diagnostics of sarcoma and provided new insight into oncogenesis. We used RNA-Seq to investigate samples diagnosed as small round cell tumors of bone, possibly Ewing sarcoma, but lacking the canonical EWSR1-ETS translocation. A new fusion was observed between the BCL6 co-repressor (BCOR) and the testis specific cyclin B3 (CCNB3) genes on chromosome X. RNA-Seq results were confirmed by RT-PCR and cloning the tumor-specific genomic translocation breakpoints. 24 BCOR-CCNB3positive tumors were identified among a series of 594 sarcomas. Gene profiling experiments indicate that $B C O R-C C N B 3$-positive cases are biologically distinct from other sarcomas, particularly Ewing's sarcoma. Finally, we show that CCNB3 immunohistochemistry is a powerful diagnostic marker for this group of sarcoma and that over-expression of BCOR-CCNB3 or of a truncated CCNB3 activates S-phase in NIH3T3 cells. Thus the intrachromosomal $X$ fusion described here represents a new subtype of bone sarcoma caused by a novel gene fusion mechanism.
\end{abstract}




\section{Text}

Sarcomas, tumors derived from the mesenchymal tissues account for approximately $2 \%$ of human cancers and constitute a very heterogeneous group of tumors that can be divided into more than 100 different subtypes on the basis of clinical, pathological, immunohistological and genetic features. In adolescents and young adults, osteosarcoma and Ewing's sarcoma (ES) are the two predominant bone sarcomas. ES is genetically characterized by gene fusions between EWSR1 and ETS members ${ }^{1-3}$. In addition, recently, EWSR1-NFATC2 fusions were observed in a minority of cases ${ }^{4}$. In osteosarcoma, various point mutations and copy number variations have been described but presently, no recurrent gene fusion has been reported ${ }^{5}$

To identify novel fusion transcripts in small round cell sarcomas we selected four cases that clinically and morphologically would be classified in the ES family of tumors yet lacked the pathognomonic gene fusions that characterize ES. They were analysed using RNA-Seq ${ }^{6-7}$ with paired-ends sequencing on a SOLiD platform. For each sample, a mean number of $123 \times 10^{6}$ paired-end (PE) reads were mapped to the reference genome (hg19). Three analysis programs, Genomatix, FusionSeq ${ }^{8,9}$ and an in-house software, that differed in their principles, were used to search for novel gene fusions. Strong fusion predictions were found in two cases. In one case, a FUSFEV putative fusion was observed and subsequently confirmed by RT-PCR (data not shown). Although not included in the gene fusion screening panel used ${ }^{10}$, this fusion was previously described in one case of $\mathrm{ES}^{11}$. In the second notable case, a total of 20 
distinct and high quality PE fragments were observed linking the $B C O R^{12}$ exon 15 and the $\mathrm{CCNB}^{13}$ exon 5 located at chrXp11.4 and chrXp11.22, respectively (Fig. 1a and b). Analysis of exon coverage showed that all BCOR exons were well represented (median base coverage around 2000) with a notable decrease of the coverage at the end of the BCOR coding sequence (median base coverage around 300) (Fig. 1c and supplementary Fig. 1). The decreased coverage at this position was specific for this tumor since it was not observed in the alignment of the three other cases (supplementary Fig. 1). For CCNB3, virtually no read was observed in the 4 first exons whereas the coverage of exon 5 to 12 was comparable to $B C O R$ (Fig. 1c).

To confirm these findings, specific primers designed in BCOR exon 15 (BCOR.1) and CCNB3 exon 5 (CCNB3.1) were used for RT-PCR experiments. The expected fragment was detected in the tumor RNA whereas no specific amplification was observed in peripheral blood cells RNA from the same patient (Fig. 1d). Sequencing of this fragment identified an in-frame fusion between the last codon of BCOR and CCNB3 exon 5 (Fig. 1e).

To further characterize the breakpoint at the genomic level, FISH experiments and long-range PCR experiments were conducted. Two BACs flanking BCOR were labeled with FITC and two BACs flanking CCNB3 were labeled with rhodamine then hybrized on nuclei from either normal cells (LL) or from case 330T. As shown on Fig. $2 \mathrm{a}$, the green or red doublets observed in normal cells were clearly disrupted in 330T nuclei with juxtaposition of green and red spots (usually appearing as yellow spots) therefore documenting the chromosome $X$ inversion. Moreover, long range PCR 
using the BCOR.1 and CCNB3.1 primers detected a specific PCR product that was observed in the tumor but not in the matched blood DNA therefore demonstrating that the rearrangement was somatically acquired (Fig. 2b). Sequencing of this fragment indicated that the fusion lies at chrX position 39,910,434 and 50,050,261 within the BCOR and MID1IP1 genes intergenic region and within the CCNB3 intron 4, respectively (Fig. 2c). Interestingly, the TGA stop codon of BCOR is included within a putative GGTGAG donor splice site sequence. Thus, the most likely hypothesis to account for this fusion mRNA is that this cryptic donor site is spliced with the acceptor site of CCNB3 exon 5, which is confirmed by cDNA junction sequence (Fig. 1e). This splicing is incomplete leading to both BCOR-CCNB3 and wild type $B C O R$ expression (Supplementary Fig. 1 \& 2).

To assess the prevalence across a large collection of sarcomas, the aforedescribed RT-PCR assay was used to screen a series of 594 sarcomas that lacked EWSR1-ETS, EWSR1-NFATC2, EWSR1-WT1, PAX3 or 7-FOXO1, the most frequent sarcoma fusions that are routinely investigated in our diagnostic laboratory. A total of 24 BCOR-CCNB3 positive cases were identified (Table 1). When tumor DNAs were available, long-range PCR confirmed the presence of genomic fusions of variable sizes (Fig. 2b \& d). Interestingly, all tumors but five were localized to bone and all occurred in adolescents or young adults. In most cases the pathological report was consistent with an undifferentiated, small round cell sarcoma, suggestive of the ES family of tumors. However, in contrast to ES, strong membrane positivity of CD99 was absent in approximately half of cases (Table 1). 
To further investigate the relationship of the BCOR-CCNB3-positive group with other sarcomas, 10 cases for which good quality RNA were available were profiled for gene expression by microarray. Resulting profiles were compared with data from osteosarcoma, rhabdomyosarcoma, synovialsarcoma, ES, neuroblastoma, malignant rhabdoid tumors and desmoplastic round cell tumors. Using a variety of unsupervised multivariate statistical factorization techniques including correspondence analysis (COA) and principal component analysis (PCA), the BCORCCNB3 group clusters distinctly from all other groups (Fig. 3a). Given the clinical and morphological similarities with the ES group, the expression profile of the BCOR-CCNB3 group was specifically compared to EWSR1-ETS-positive ES and our findings confirmed the striking difference between the two groups with more than 3000 probesets showing highly significant differences of expression levels (Fig. 3b). The BCOR-CCNB3/ES comparison was used for a gene set enrichment analysis. Two of the most significantly different gene sets corresponded to Ewing specific gene sets including the Zhang_Targets_of_EWSR1_FLI1_fusion and the Riggi_Ewing_sarcoma_progenitor signatures (Fig. 3 c \& d; Supplementary Fig. 3 \& 4). In particular, most of the well-known EWSR1-FLI-1 target genes ${ }^{3}$, like NR0B1, CAV $1 \mathcal{E}$ 2, PRKCB, NKX2-2, IGFBPS, CCK, MYC, which have been shown to play critical roles in ES oncogenesis were not expressed in the BCOR-CCNB3 tumors.

Finally, SNP6.0 arrays detected no alteration in 11 cases out of 18 studied cases. In the other cases, the only recurrent abnormalities were chromosome 17p and 10q deletions, each observed in two cases. Neither deletion nor amplification of the 
fusion partners was observed. Importantly, the most frequent copy number abnormalities of $\mathrm{ES}^{14}$, i.e. chromosome 8 gain that is observed in approximately $50 \%$ of ES cases, chromosome gain of $1 \mathrm{q}$ and 12 or chromosome $16 \mathrm{q}$ loss that are each observed in approximately $25 \%$ of ES cases were not recurrent characteristics of $B C O R-C C N B 3$ positive cases. These data indicate that the classical ES and BCORCCNB3 tumors do not share common copy number changes. Altogether, these data show that a new group of bone sarcomas is characterized by a recurrent $B C O R$ CCNB3 fusion as a result of a chromosome $\mathrm{X}$ paracentric inversion. Despite remarkable clinical and pathological similarities with the group of ES, gene profiling and SNP-array experiments indicate that this group of tumors is biologically distinct from ES and, in particular, does not share the EWSR1-ETS signature of ES. We propose to identify this new group as a distinct type of Ewing-like tumors. To further investigate key oncogenic pathways that may characterize the BCOR-CCNB3-positive cases, a differential analysis was conducted between the BCOR-CCNB3-positive group and other tumor types (see Fig. 5; to avoid over-representation of any tumor type in the analysis the comparison was made using 10 cases randomly selected among each of the 11 tumor types)(supplementary Tables 1-3). Gene set enrichment analyses (GSEA) as well as DAVID pathway analyses indicated that morphogenesis and development, in particular of the skeletal system, showed up very strongly due to the over-expression of numerous homeobox genes. Even more importantly, activation of the WNT and SHH signaling pathways also appeared highly significant in this analysis (Fig. 4a, supplementary Tables 4-6). 
It is notable that the newly characterized rearrangement includes two important genes on the $\mathrm{X}$ chromosome. BCOR encodes a ubiquitously expressed transcriptional repressor that associates with the BCL6 oncoprotein and with a variety of histone modifying enzymes suggesting that it acts as suppressor of gene expression through epigenetic mechanisms ${ }^{12,15}$. Of particular interest regarding its involvement in bone tumors is the observation that $B C O R$ regulates mesenchymal stem cell function by epigenetic mechanisms ${ }^{16}$. BCOR has been associated with human diseases. Indeed, mutations of $B C O R$ are responsible for the inherited oculofaciocardiodental and Lenz microphtalmia syndromes ${ }^{17}$. Moreover, $B C O R$ has recently been described as a fusion partner of RARalpha in acute promyelocytic leukemia and as a putative tumor suppressor gene in a subset of acute myeloid leukemia $^{18,19}$.

CCNB3 exhibits a pattern of expression highly restricted to the testis and is an early meiotic cyclin that is expressed in leptotene and zygotene phases of spermatogenesis $^{13,20}$. This strongly suggests that ectopic expression of CCNB3 as a result of the $B C O R$ gene fusion may constitute a key oncogenic event in this newly characterized type of Ewing-like tumors. This was further investigated by BrdU staining after over-expressing $B C O R-C C N B 3$ or deltaCCNB3, a truncated version of CCNB3 corresponding to the portion involved in the fusion gene, in mouse NIH3T3 fibroblasts. As compared to the empty vector, both expression vectors led to a twofold increase of the amount of cells in S-phase (Fig. 4b and supplementary Fig. 6). Read counts (Figure 1c) and microarray experiments that show similar intensities for 
the expression of 5' BCOR and 3'CCNB3 moieties (Fig. 4a and supplementary Fig. 2) strongly suggest that $B C O R-C C N B 3$ is expressed as a full transcript and that the truncated CCNB3 is not expressed per se. Though cell cycle analyses clearly indicate that the CCNB3 portion of the fusion is potentially sufficient to mediate cell cycle effects, it is also expected that oncogenesis relies on additional phenotypic effects of the full size fusion protein.

Finally, in the series of close to 1000 tumor samples profiled in our laboratory including a spectrum of pediatric tumors, adult sarcomas and brain tumors, only the $B C O R-C C N B 3$-positive cases exhibited a very high expression level of the CCNB3 probesets indicating the outstanding specificity of this marker (Fig. 5a). This specificity was further tested using a CCNB3 specific antibody. A total of 43 tumor samples was investigated including $B C O R-C C N B 3$-positive cases, Ewing sarcomas, small round cell desmoplastic tumors, rhabdomyosarcomas, synovial sarcomas. In contrast to the 25 BCOR-CCNB3-negative cases that stained completely negative, the 18 BCOR-CCNB3-positive cases that could be tested exhibited strong nuclear CCNB3 staining (Fig. 5b). This therefore highlights the potential of a simple CCNB3 immunohistochemistry assay to be used as a diagnostic test for this subgroup of sarcoma.

In conclusion, our study highlights the power of next generation sequencing, particularly RNA-Seq, and microarray experiments for the refined sub-classification of the highly heterogeneous group of sarcomas. Our results have uncovered a distinct gene fusion that shed new light on the development of two tumors with 
similar phenotypes, Ewing and Ewing-like tumors. In this regard, we can infer distinct mechanisms of oncogenesis, an observation of critical significance for both diagnostic and possibly, therapeutic interventions in the coming age of personalized medicine. Possible relationships of this group of tumors with CIC-DUX4-positive cases will also be important to consider ${ }^{21}$. Further studies will be required to model the BCOR-CCNB3 fusion in cell and animal models in order to decipher the downstream consequences of this fusion, evaluate the respective contributions of the $B C O R$ and $C C N B 3$ portions of the fusion and gain insights into the underlying oncogenic processes. 


\section{Accession Numbers}

The BCOR-CCNB3 sequence has been deposited in GeneBank (accession number JN813375) and Affymetrix expression data in Gene Expression Omnibus (GSE34800)

\section{Acknowledgements}

We thank Stephen Chanock for fruitful discussions and critical reading of the manuscript. We are indebted to Virginie Raynal, Patricia Legoix-Ne, Alain Nicolas, David Gentien, Séverine Lair, Alban Lermine, Emmanuel Barillot for critical technical help. We thank the following individuals for contributing cases and paraffin blocks or for supplying clinical information: J Champigneulle, A Croue, $\mathrm{P}$

Dechelotte, JM Guinebretière, C Jeanne-Pasquier, A Moreau, JM Picquenot, D Ranchère-Vince, X Sastre-Garau, P Terrier, MC Vacher-Lavenue, V Verkaere, L Guillou. We thank Emmanuel Louis, Samuel Thoraval and Thierry Scarcez from the Life Science Company for help in applying the SOLiD technology. We also thank Nancy Bretschneider, Claudia Gugenmus, Mattias Scherf and Martin Seifert from the Genomatix company for the analysis of data.

This work was supported by grants from the Ligue Nationale Contre le Cancer (Equipe labellisée and CIT program), the Région Ile de France, the INCa ( $\mathrm{n}^{\circ} 2008-044$, 0627 and ZP09-027-EPI), the European Union (EET pipeline), and the following associations: Courir pour Mathieu, Dans les pas du Géant, Olivier Chape, Les

Bagouzamanon and les Amis de Claire. 


\section{Author contributions}

SB performed the NGS analysis; GP, SR, and SB characterized the initial case and screened the sarcoma patients; CL and FT performed all the bioinformatics analyses; JMC performed pathological review of the cases and CCNB3 immunohistochemistery experiments; SCG contributed the analysis of clinical files from patients, and with VP to the cloning and cell cycle analyses of BCOR-CCNB3 and deltaCCNB3 cDNAs; GP, FT and OD planned and supervised the work and all authors contributed writing the manuscript.

\section{Competing financial interests}

The authors declare no competing financial interests. 
Table 1. Clinical and pathological data of patients harboring BCOR-CCNB3 Ewing-like tumors

\begin{tabular}{|c|c|c|c|c|c|c|}
\hline $\begin{array}{l}\text { Case } \\
\text { no. }\end{array}$ & $\begin{array}{c}\text { Age at } \\
\text { diagnosis } \\
\text { (yrs) }\end{array}$ & Gender & Tumor site & Pathology $y^{a}$ & CD99b & $\begin{array}{c}\text { Follow- } \\
\text { up }^{c}\end{array}$ \\
\hline 330 & 15 & $\mathrm{M}$ & femur & SRCS & $\mathrm{M}$ & $\mathrm{MR} / \mathrm{DOD}$ \\
\hline 985 & 6 & $\mathrm{~F}$ & neck* & SRCS & M & $\mathrm{CR}$ \\
\hline 986 & 13 & $\mathrm{~F}$ & femur & SRCS & M & $\mathrm{U}$ \\
\hline 987 & 14 & M & toe & FCS & $\mathrm{C}$ & $\mathrm{MR} / \mathrm{DOD}$ \\
\hline 988 & 13 & M & femur & SRCS & $\mathrm{U}$ & MR \\
\hline 991 & 6 & $\mathrm{~F}$ & vertebra & SRCS & C & $\mathrm{U}$ \\
\hline 992 & 15 & M & tibia & SRCS & - & $\mathrm{CR}$ \\
\hline 993 & 13 & M & clavicle & SRCS & M & $\mathrm{U}$ \\
\hline 994 & 11 & M & para-vertebral ${ }^{*}$ & SRCS & $\mathrm{C}$ & $\mathrm{U}$ \\
\hline 995 & 26 & $\mathrm{~F}$ & chest wall* & SRCS & - & $\mathrm{U}$ \\
\hline 996 & 12 & M & pubis & FCS & C & $\mathrm{CR}$ \\
\hline 997 & 10 & M & talus bone & SRCS & M & MR/DOD \\
\hline 1005 & 13 & M & sacrum & U & $\mathrm{U}$ & U \\
\hline 1006 & 22 & M & pelvis* & SRCS or SV & M & DOD \\
\hline 1007 & 19 & M & rib & SRCS & M & $\mathrm{CR}$ \\
\hline 1008 & 14 & M & iliac crest & $\mathrm{SCO}$ & $\mathrm{C}$ & $\mathrm{CR}$ \\
\hline 1009 & 17 & $\mathrm{~F}$ & sacrum & $\mathrm{U}$ & - & $\mathrm{U}$ \\
\hline 1010 & 22 & M & iliac crest & $\mathrm{SCO}$ & M & $\mathrm{CR}$ \\
\hline 1011 & 8 & $\mathrm{~F}$ & vertebra & Granulocytic sarcoma & $\mathrm{U}$ & DOD \\
\hline 1012 & 19 & $\mathrm{~F}$ & abdominal wall $^{*}$ & Sarcoma or MPNST & C & $\mathrm{CR}$ \\
\hline 1013 & 13 & M & femur & FCS & C & DOD \\
\hline 1014 & 13 & $\mathrm{~F}$ & sacrum & MPNST or FCS or PNET & C & OT \\
\hline 1015 & 20 & M & iliopubic branch & $\mathrm{U}$ & $\mathrm{U}$ & $\mathrm{U}$ \\
\hline 1016 & 9 & M & femur & ESFT/ PNET & U & DOD \\
\hline
\end{tabular}

a: SRCS, small round cell sarcoma; FCS, Fusiform cell sarcoma; SV, Synovialosarcoma; MPNST, malignant peripheral nerve sheet tumor; SCO, small cell osteosarcoma; ESFT, Ewing sarcoma family of tumors; PNET, Primitive neuroectodermal tumor; U, Unknown.

b: $M$, membrane positivity; $C$, cytoplasmic positivity; -, negative; $U$, unknown.

c: MR, metastatic relapse; DOD, dead of disease; CR, complete remission; LR, local relapse; OT, ongoing treatment; $\mathrm{U}$, unknown status.

${ }^{*}$ soft tissue tumour 


\section{Legends to figures}

\section{Figure 1. RNA-Seq identification of the BCOR-CCNB3 fusion.}

a - Scheme of the paired-end coverage of the junction between BCOR and CCNB3 sequences using IGVtools. The transcription orientation is reported in the $5^{\prime}-3^{\prime}$ direction from $B C O R$ exon 15 to $C C N B 3$ exon5. The junction between both genes is represented by the vertical dotted line. Each PE read is represented by a horizontal segment consisting of an orange box on the left end (the 50bp F3 mate) and a grey box on the right end (the F5 25bp mate) connected by a line representing the unsequenced part of the PE fragment. Duplicated reads have been removed according to the criteria that only PE reads having F3 mates starting at distinct positions are conserved. F3 reads were aligned 5' to $3^{\prime}$ along the $B C O R$ sequence.

$\mathrm{b}$ - A scheme of chromosome $\mathrm{X}$ with positions and strand orientations of the $B C O R$ and CCNB3 genes.

c - Read coverage of the BCOR and CCNB3 exons (BioScope alignement). The base coverage corresponding to each intron or exon sequence is indicated on top of the genomic organization of $B C O R$ and $C C N B 3$ genes (the $\mathrm{Y}$ axis corresponds to the scale of the base coverage). The median base coverage including duplicates of BCOR exons is 2000 (maximum 3939 in exon 4), comparable to CCNB3 coverage (median 2500, maximum 5394 on exon 5).

d - RT-PCR detection of a specific 290 base-pair fragment in tumor RNA (T) but not in blood RNAs (C) from the same patient (case 330). CTL: PCR negative control. L: 100bp ladder. 
e - Structure of the BCOR-CCNB3 cDNA. The three ankyrin domains of BCOR are indicated in purple and the two cyclin domain of CCNB3 in green. The sequence of the $B C O R-C C N B 3$ junction together with the encoded peptide sequence is reported below. The complete BCOR-CCNB3 nucleotide and peptide sequences are shown in Supplementary Fig. 5.

Figure 2. Characterization of the $B C O R-C C N B 3$ genomic rearrangement and demonstration of its recurrence in small round cell sarcomas

a - A chromosome $\mathrm{X}$ paracentric inversion is documented by FISH experiments. BACs RP11-91I16 and RP11-66502 were labeled with FITC. BACs CTD-2107N14 and RP11-576P23 were labeled with rhodamine. The arrows indicate position and orientation of the BCOR and CCNB3 genes. In normal cells (lymphoblastoid cell line, LL), the two red spots (or the two green spots) are in close vicinity and appear either as a single spot or as unicolor doublets. In BCOR-CCNB3-positive cells (330T cells), following the chromosome $\mathrm{X}$ paracentric inversion, the two red and green spots are split and appear as bicolored doublets. Scale bar represent $1 \mu \mathrm{m}$.

b - Left panel: long-range PCR amplification of a specific fragment in the tumor DNA (T) but not in the constitutional DNA (C) of case 330. Right panel: long range PCR of various sizes on genomic DNAs from RT-PCR-positive BCOR-CCNB3 cases. CTL, PCR negative control. L: 1Kb ladder.

c - Genomic organization of the BCOR-CCNB3 fusion gene in case 330. A focus on the breakpoint is shown below. BCOR and CCNB3 sequences are indicated in orange and 
black respectively. The TGA stop codon of BCOR is underlined. As can be seen from the comparison of the genomic and cDNA sequences (Fig. 1e), the BCOR TGA stop codon is embedded in a GGTGAG sequence that is used as a donor site spliced with the CCNB3 exon 5 acceptor site.

$\mathrm{d}$ - Position of the breakpoints within the BCOR 3' UTR or BCOR/MID1IB1 intergenic region and within the CCNB3 intron 4 region in different $B C O R-C C N B 3$-positive samples.

Figure 3. BCOR-CCNB3 positive cases are distinct from other small round cell tumors and particularly from Ewing's sarcoma.

a- An unsupervised COA multivariate analysis comparing BCOR-CCNB3-positive cases with desmoplastic small round cell tumor (DSRCT), Ewing's sarcoma (ES) and malignant rhabdoid tumor cases (MRT). Ostesarcoma, neuroblastoma, rhabdomyosarcoma and synovial sarcoma are not shown for the clarity of the figure. Ewing's sarcoma profiled in the same experiment as BCOR-CCNB3 are indicated in green and Ewing's sarcomas profiled on the same platform but in previous experiments are indicated in blue. This indicates that the distinct clustering of $B C O R$ CCNB3 positive cases and Ewing sarcomas is not linked to a platform bias.

b- A volcano plot comparing BCOR-CCNB3 positive cases with EWSR1-ETS Ewing's sarcoma positive cases. Expression levels were compared using the Welch t-test. Probesets with Bonferroni adjusted Welch two sample t-test $P$ values $<0.01$ and $\log$ 
ratio of mean values between the BCOR-CCNB3 and ES groups $>3$ are indicated in red (3203 probesets present these characteristics).

c- GSEA on genes ranked differentially between BCOR -CCNB3 positive cases and EWSR1-ETS positive Ewing's sarcomas. Bars indicate each gene of the Zhang_targets_of_EWSR1_FLI1_fusion signature. A strong anti-correlation is observed between BCOR-CCNB3 overexpressed genes and the EWSR1-ETS signature.

d- Heatmap of the EWSR1-FLI1 signature in BCOR-CCNB3 positive cases. The genes were ranked using signal-to-noise metric. A complete annotation of this figure is provided in supplementary Fig.3. Similar results were obtained with the gene set RIGGI_Ewing_sarcoma_progenitor_up signature (Supplementary Fig. 3 \& 4).

\section{Figure 4. Functional consequences of BCOR-CCNB3 expression.}

a- GSEA on genes ranked differentially between BCOR-CCNB3-positive cases and other sarcomas or pediatric tumors (10 tumors randomly chosen in each of the 11 tumor groups shown in Fig. 5a) identifies signatures of Hedgehog and WNT pathways activation (supplementary Table 6).

b- Increased S-phase associated with BCOR-CCNB3 expression. NIH3T3 cells were transfected with BCOR-CCNB3 or truncated CCNB3 (deltaCCNB3) and cell cycle was analysed after BrdU staining (n=3; Welch two sample t-test $P$ values=3.3 $10^{-3}$ and 2.4 $10^{-3}$ as compared to empty vector, respectively). 


\section{Figure 5. Expression of CCNB3 across a series of tumors.}

a - Boxplots of the intensity of the 231481_at probeset (log scale) across the BCORCCNB3 positive cases (10 cases), desmoplastic small round cell tumors (DRSCT, EWSR1-WT1 positive, 32 cases), Ewing sarcomas (ES , EWSR1-ETS positive, 74 cases), gliomas (17 cases), medulloblastomas (Mb, 52 cases), SMARCB1 deficient Malignant rhabdoid (MRT, 35 cases), neuroblastomas ( $\mathrm{Nb}, 64$ cases), osteosarcomas (OS, 35 cases), retinoblastomas (Rb, 39 cases), rhabdomyosarcomas (RMS, 121 cases), synovial sarcomas (Synov, 34 cases), and other sarcomas (288 cases). Normal includes 353 normal tissues (GSE3526) of which three testis samples corresponding to the upper outliers. The sarcomas that express intermediate levels of CCNB3 tested negative for the BCOR-CCNB3 fusion. The expression level of BCOR is reported in supplementary Fig.2.

b - Hematoxylin-Eosin- staining (HES), immunohistochemistry for CD99 and CCNB3 in an EWSR1-FLI-1-positive Ewing sarcoma as compared to two BCORCCNB3- positive cases. Scale bar (black line) is $20 \mu \mathrm{M}$ for all panels except for CCNB3 staining of BCOR-CCNB3-positive cases where it is $40 \mu \mathrm{M}$ to highlight the negative staining of stromal components (white arrows). 


\section{Online methods}

\section{RNA extraction}

Tumors were snap frozen in liquid nitrogen. After crushing tumors, total RNA was isolated using the Trizol reagent kit (Invitrogen, Carlsbad, Ca) based on the GITC phenol chloroform extraction method. RNAs were quantified by Qubit (Invitrogen, Carlsbad, Ca) and Nanodrop ND1000 (ThermoFisher Scientific, Waltham, MA) before quality assessment with the Agilent 2100 Bioanalyzer (Santa Clara, Ca).

RNA library construction

PolyA mRNAs were purified from $10 \mu \mathrm{g}$ of total RNA using NucleoTrap ${ }^{\circledR}$ mRNA (Macherey Nagel, Düren, Deutschland) according to the manufacturer's protocol.

Following the SOLiD TM Total RNA Seq Kit instructions, 100 ng of polyA RNAs were fragmented by incubation with RNAse III for $10 \mathrm{~min}$ in a $10 \mu$ reaction volume containing 1 X RNase III buffer supplied with the enzyme. Fragmented RNAs were then purified using the RiboMinus ${ }^{\mathrm{TM}}$ Concentration Module (Invitrogen, Carlsbad, Ca). The yield and size distribution of the fragmented RNA were assessed using the Quant-iT ${ }^{\mathrm{TM}}$ RNA assay kit with the Qubit® fluorometer (Invitrogen, Carlsbad, Ca) and the RNA 6000 Pico Chip Kit with the Agilent® 2100 Bioanalyzer (Agilent, Santa Clara, Ca). A total of $50 \mathrm{ng}$ of fragmented RNAs were hybridized and ligated with the SOLiD ${ }^{\mathrm{TM}}$ adaptator mix and reverse transcribed according to supplier instructions. 
The isolated cDNA were size selected around 200 bp using Novex® pre-cast gel products. The cDNA were then amplified according to the SOLiD TM Total RNA Seq Kit. The yield and size distribution of the cDNA were assessed using the QuantiT ${ }^{\mathrm{TM}}$ HS DNA assay kit with the Qubit ${ }^{\circledR}$ fluorometer (Invitrogen, Carlsbad, Ca) and the High Sensitivity DNA Assay Chip Kit on the Agilent ${ }^{\circledR} 2100$ Bioanalyzer.

Emulsion PCR

Templated beads were generated for sequencing using standard manufacturer protocols. Briefly, an aqueous phase was prepared from the SOLiD ePCR kit containing AmpliTaq Gold DNA Polymerase UP, buffer, $\mathrm{MgCl}$, dNTPs, amplification primers and library template. The aqueous phase was then introduced to an oil phase in an ULTRA-TURRAX Turbo Drive (IKA) to create a water-in-oil emulsion. The emulsion was then transferred to a 96 well plate and PCR amplified using the recommended PCR conditions. After PCR amplification, emulsions were broken using butanol, and the beads were washed, enriched, and treated with terminal transferase before quantification and deposition onto a slide for sequencing. Whole Transcriptome paired end sequencing

Templated beads were deposited on two quadrants of a slide per sample. Massively parallel ligation sequencing was carried out using Applied Biosystems SOLiD System (V4 chemistry) following supplier instructions.

\section{NGS analysis}


Apart from Genomatix analysis NGS raw data was aligned in color space using the color-code error-correction capability of LifeTech's BioScope environment and a set of human genome reference sequences associated to the hg19 version of Human Genome. The distribution of insert size, excluding adaptors was 116bp (90\% confidence interval 60-163bp). NGS analysis was performed using three independent Paired-End (PE) Gene Fusion analysis pipelines: Genomatix NGS, by Genomatix Software $\mathrm{GmbH}$ computational biology company (http://www.genomatix.de), FusionSeq, by the Gerstein academic laboratory (http://rnaseq.gersteinlab.org/fusionseq/) $)^{1}$ and an in-house pipeline. All three methods, although quite different in conception, detected successfully the presence of a novel BCOR/CCNB3 chimeric fusion in one sample (330T) and the known FUS/FEV fusion in another sample.

\section{Genomatix NGS pipeline}

Alignment and analysis of the NGS datasets were performed directly by Genomatix bioinformaticians (http://www.genomatix.de). Raw data were aligned against the human genome (NCBI_build 37) and transcriptome (ElDorado 12-2010; containing transcript annotation from RefSeq, GeneBank, Ensembl and crossmappings) using the two step mapping strategy from the Genomatix Mining Station. Data were mapped with low stringency allowing one point mutation in the seed search (deep) and 85\% overall alignment quality in the subsequent step. In addition, global and local spliced alignment was performed to find split reads covering exon boundaries and fusion events. PE statistics were calculated 
automatically (mean insert size, standard deviation, strand orientation and pileup distribution) and subsequently used for gene fusion detection.

Gene fusions within and across chromosomes were derived from uniquely mapped PE reads in four steps including filtering, clustering, inclusion of splicejunction information, and scoring. The filtering removed read pairs if one or both reads did not uniquely map to the genome, if the reads aligned within the expected distance, or if any pair was contained in an artificial pileup. All PE that pass the filters and were mapped to transcripts from different loci were considered as gene fusion candidates. After clustering reads mapping to proximal positions, global spliced-alignments were included as an additional line of evidence to determine the exact breakpoint. In the last step, the gene fusion candidates were ranked. The two scores are the number of spliced reads and the number of PE fragments that support any gene fusion candidate (supplementary Table 7).

\section{FusionSeq pipeline}

NGS raw data was aligned in color space using the color-code error-correction capability of LifeTech's BioScope environment. Overall the alignment was ungapped allowing 2 color code mismatches against the reference sequence for each PE read. Local clipping of $<5$ bases at the start or the end of the PE reads were allowed to maximize the number of aligned reads. In first instance, the F3 and F5 PE mates were aligned independently against three hg19 reference libraries: a library of non-coding sequences (784 non-coding sequences delivered with the BioScope software (including $\mathrm{SOLiD}^{\mathrm{TM}} 4$ adapter sequences, rRNAs, tRNAs, Single-base-repeat 
sequences, including Poly-A, Poly-T...) to be discarded during alignment, the human genome reference library and a canonical splice junction library, built from RefSeq annotations (.gtf file available via UCSC) that reconstitute the junction sequence between two consecutive exons.

The results of F3 and F5 read alignments were then merged and pairing was performed. No quality filter was applied in advance and the resulting BAM file held the complete set of aligned PE reads.

The BAM file was then passed to FusionSeq following the authors instructions (http://rnaseq.gersteinlab.org/fusionseq/) ${ }^{9}$. The fusion transcript detection module employs the UCSC known Genes set that contains 66,803 isoforms. First, poor quality and orphan reads were removed. Then PE reads that mapped to the same gene were considered as part of the normal transcriptome. PE reads that mapped to different genes were selected as potential candidate fusion transcripts. The complete list of fusion proposals was then passed through a "filtration cascade" to remove spurious candidate fusion genes. We applied in the order the "MitochondrialFilter" and "RepeatMaskerFilter" procedures. The "AnnotationConsistencyFilters" was also applied to remove Fusion Gene candidates where one of the partners was annotated as "collagen", "clone", "ribosomal" or "pseudogene", which were supposed to have elevated sequence homology with genes of the same class. The reduced list of fusion proposals was then classified by FusionSeq according to the type of genomic region involved in a fusion candidate (exons, introns, splice junctions or UTR regions), the absolute number of Exon-to-Exon PE reads encompassing the junction and the 
following three statistical indicators: normalized number of Supportive Paired-End Reads (SPER), Difference between the Analytically calculated and Expected SPER (DASPER) and the Ratio of Empirically computed SPERs (RESPER). We extracted from this conspicuous data-set the information concerning the Exon-to-Exon junctions. We used the absolute number of PE fragments encompassing the fusion junction and the DASPER indicator to rank the fusion proposals. This procedure returned a list of 16 fusion proposals that are reported in supplementary Table 8 . Probable False-positive candidates were further filtered out based on 1-mapping quality (MAPQ>=20), 2- non redundancy (only one PE was retained for groups of PE reads with F3 and F5 mates starting at identical positions), 3- length of F3 and F5 reads (only 50 and 25bp length mates were retained), 4- consistency of insert size assuming an intronic breakpoint (insert size in the range 60 to 163 was considered normal) and 5- unambiguous mapping (PE reads having homologous sequences in exome and genome were discarded)(Supplementary Table 8).

Inhouse pipeline

A set of in-house scripts was written in bash, awk and $\mathrm{R}$ language for Linux system. Raw data was aligned in color space using BioScope ungapped alignment modules but a CDS library for each gene was used instead. CDS annotations were extracted from the UCSC RefSeq GTF annotation file and a fasta seq reference library was built by concatenating the sequence of each exon in their genomic order for each gene. F3 and F5 PE reads were aligned independently by allowing 2 color code mismatches for each read. Finally, the Read-Pairing module of Bioscope was used to 
create a PE BAM file. A first read quality filter was applied to remove PE reads whose "mapq" score from samtools quality indicator was lower than 20. We then separated PE reads contained within a single gene from those encompassing different genes by simply checking the gene partners, orientation and expected insert size associated in each PE fragment. We picked the top 10 fusion gene proposals with the higher number of PE read encompassing the junction (supplementary Table 9). False positive candidates were further filtered out using the five criteria aforementioned for the FusionSeq pipeline.

RT-PCR and long range genomic PCR experiments

BCOR3.1 and CCNB3.1 primers (Supplementary Table 10) were used to amplify cDNA and genomic sequences.

\section{Microarray data}

Experimental procedures for GeneChip microarray were performed according to the Affymetrix GeneChip Expression Analysis Technical Manual (Affymetrix, Santa Clara, CA) using HG-U133-Plus2 arrays. All microarray data were simultaneously normalized using the gcrma package version 2.22.0 in R 2.12.0 environment ${ }^{22}$ and quality assessment was based on Relative Log Expression and Normalized Unscaled Standard Errors (http://www2.warwick.ac.uk/fac/sci/statistics/staff/academicresearch/brettschneider). COA analysis was performed using made $4^{23}$ and $\operatorname{rgl}$ (developed by Daniel Adler and Duncan Murdoch) R packages. Gene set enrichment analysis ${ }^{24}$ was done by comparing Ewing-like and ES cases using Signal / Noise ratio algorithm. Ewing-like versus ES comparison was performed using the Welsh t-test 
and associated $\mathrm{P}$ values were corrected for multiple testing with the Bonferroni method. SAM analyses were performed with the samr $\mathrm{R}$ package ${ }^{22,25}$ using the 10 BCOR-CCNB3-positive samples with a random selection of 10 samples in each 11 other tumor types. Ten different analyses were performed with different samplings. Deltas were chosen as first value with 90th percentile FDR below 0.01. DAVID analyses $^{26}$ were performed online (http://david.abcc.ncifcrf.gov/home.jsp) using EASE value $<10^{-5}$, count $>10$, Fold Enrichment $>2$ and Bonferroni $P$ value $<10^{-2}$ parameters.

\section{FISH experiments}

FISH analysis were performed on $4 \mu \mathrm{m}$ frozen tissue section fixed $10 \mathrm{~min}$ in $1 / 3 \mathrm{v} / \mathrm{v}$ Acetic Acid/Ethanol bath. The slides were immersed in 2X SSC pH 7.0 for $30 \mathrm{~min}$ at $37^{\circ}$. The sections were then sequentially dehydrated in alcohol $(70 \%, 90 \%$ and $100 \%)$ for 2 min each and air dried. The BCOR probe was composed of a set of two flanking BAC clones (CHORI): RP11-91I16 (telomeric) and RP11-665O2 (centromeric) both biotin labeled (Biotin Nick translation mix, Roche) and Avidin-Fluorescein revealed (Roche). The CCNB3 flanking probes were CTD- 2107N14 (telomeric) and RP11576P23 (centromeric) both digoxigenin labeled (DIG Nick translation mix, Roche) and anti-digoxygenin-Rhodamine Fab Fragment detected (Roche). All BAC positions were assessed by blat after SP6 and T7 end sequencing.

The probes were validated on normal metaphase (male patient), each locus showing an unambiguous unique green or red signal localized on $X$ chromosome.

Immunohistochemistry 
$4 \mu$ m-thick paraffin sections were cut and mounted on glass slides (Superfrost+ Menzel Glazer). Subsequently the preparations were entirely treated on automate VENTANA-Benchmark-XT®. The primary antibody was a CCNB3 polyclonal anti rabbit antibody from Sigma Aldrich (St Quentin Fallavier France, Ref HPA000496) and was used at a 1:200 dilution.

BCOR-CCNB3 fusion cDNA cloning

cDNA from case 330T was PCR amplified with Phusion DNA polymerase (Finnzymes, Fisher Scientific, Illkirch, France) and EcoRV_BCOR_fw and XhoI_CCNB3_rev (Supplementary Table 10) primers. EcorV/Xho1 digested fragment was then ligated into pCDNA3.1(+) vector (Invitrogen, Cergy-Pontoise, France) between EcoRV and XhoI restriction sites. DeltaCCNB3 was PCR amplified from cDNA obtained from testis mRNA (OriGene Technologies, USA) using EcoRV_DCCNB3_fw and XhoI_CCNB3_rev primers (Supplementary Table 10) and cloned into pcDNA3.1(+) vector. The complete cDNA sequences were checked. Cellcycle analyses were performed by BrdU incorporation as previously described ${ }^{27}$. 


\section{REFERENCES}

1- Delattre, O. et al. Gene fusion with an ETS domain caused by chromosome translocation in human tumors. Nature. 359, 162-165 (1992).

2- Delattre, O. et al. The Ewing family of tumors: a subgroup of small round cell tumors defined by specific chimeric transcripts. New Engl J Med. 331, 294-299 (1994). 3-Toomey, E.C., Schiffman, J.D., Lessnick SL. Recent advances in the molecular pathogenesis of Ewing's sarcoma. Oncogene. 29, 4504-16 (2010).

4-Szuhai, K. et al. The NFATc2 gene is involved in a novel cloned translocation in a Ewing sarcoma variant that couples its function in immunology to oncology. Clin Cancer Res. 15, 2259-68 (2009).

5- Broadhead, M.L.,Clark, J.C., Myers, D.E., Dass, C.R. The molecular pathogenesis of osteosarcoma: a review. Sarcoma. 2011, 959248 (2011).

6- Wilhem, B.T. et al. Dynamic repertoire of a eukaryotic transcriptome surveyed at single-nucleotide resolution. Nature 453, 1239-43 (2008)

7-Mortazavi, A., Williams, B.A., McCue, K., Schaeffer, L., Wold, B. Mapping and quantifying mammalian transcriptomes by RNA-Seq. Nat Methods. 5, 621-8 (2008).

8- Maher, C.A. et al. Chimeric transcript discovery by paired-end transcriptome sequencing. Proc Natl Acad Sci U S A. 106, 12353-8. (2009)

9- Sboner, et al. FusionSeq: a modular framework for finding gene fusions by analyzing paired-end RNA-sequencing data. Genome Biol. 11, R104 (2010) 
10- Peter, M., Gilbert, E., Delattre, O. A multiplex real-time pcr assay for the detection of gene fusions observed in solid tumors. Lab Invest. 81, 905-12 (2001).

11- $\mathrm{Ng}$, T.L et al. Ewing sarcoma with novel translocation $\mathrm{t}(2 ; 16)$ producing an inframe fusion of FUS and FEV. J Mol Diagn. 9, 459-63 (2007)

12- Huynh, K.D., Fischle, W., Verdin, E., Bardwell, V.J. BCoR, a novel corepressor involved in BCL-6 repression. Genes Dev. 14, 1810-23 (2000).

13- Gallant, P., Nigg, E.A. Identification of a novel vertebrate cyclin: cyclin B3 shares properties with both A- and B-type cyclins. EMBO J. 13, 595-605 (1994).

14- Armengol, G. et al. Recurrent gains of 1q, 8 and 12 in the Ewing family of tumours by comparative genomic hybridization. Br J Cancer 75,1403-9 (1997)

15- Gearhart, M.D., Corcoran, C.M., Wamstad, J.A., Bardwell, V.J. Polycomb Group and SCF Ubiquitin Ligases Are Found in a Novel BCOR Complex That Is Recruited to BCL6 Targets Mol Cell Biol, 26, 6880-6889 (2006)

16- Fan, Z. et al. BCOR regulates mesenchymal stem cell function by epigenetic mechanisms. Nat Cell Biol. 11, 1002-9 (2009).

17- Ng, D. et al. Oculofaciocardiodental and Lenz microphthalmia syndromes result from distinct classes of mutations in BCOR. Nat Genet. 36, 411-6 (2004).

18- Yamamoto, Y. et al. BCOR as a novel fusion partner of retinoic acid receptor alpha in a $\mathrm{t}(\mathrm{X} ; 17)(\mathrm{p} 11 ; \mathrm{q} 12)$ variant of acute promyelocytic leukemia. Blood 116, 4274-83 (2010).

19- Grossmann et al. Whole genome sequencing identifies somatic mutations of BCOR in acute myeloid leukemia with normal karyotype. Blood 118, 6153-63 (2011). 
20- Nguyen, T.B. et al. Characterization and expression of mammalian cyclin b3, a prepachytene meiotic cyclin. J Biol Chem. 277, 41960-9 (2002).

21- Kawamura-Saito et al. Fusion between CIC and DUX4 up-regulates PEA3 family genes in Ewing-like sarcomas with $\mathrm{t}(4 ; 19)(\mathrm{q} 35 ; \mathrm{q} 13)$ translocation. Hum. Mol. Genet. $15,2125-37$ (2006).

22- The R manual. Venables, W.N. \& Smith, D.M. R Development Core Team, ed. (2005). R: A language and environment for statistical computing. (Vienna, Austria.). 23- Culhane, A. C., Thioulouse, J., Perriere, G., and Higgins, D. G. MADE4: an R package for multivariate analysis of gene expression data. Bioinformatics (Oxford, England) 21, 2789-2790 (2005)

24- Subramanian, A., et al., Gene set enrichment analysis: a knowledge-based approach for interpreting genome-wide experssion profiles, Proc Natl Acad Sci U S A $102,15545-50(2005)$

25- Tusher, V.G., Tibshirani, R. \& Chu, G. Significance analysis of microarrays applied to the ionizing radiation response. Proc. Natl. Acad. Sci. U.S.A. 98, 5116-21 (2001)

26- Huang DW, et al. Bioinformatics enrichment tools: paths toward the comprehensive functional analysis of large gene lists. Nucleic Acids Res. 37, 1-13

27- Dauphinot et al. Analysis of the expression of cell cycle regulators in Ewing cell lines: EWS-FLI-1 modulates p57KIP2and c-Myc expression. Oncogene 20, 3258-65 (2001) 


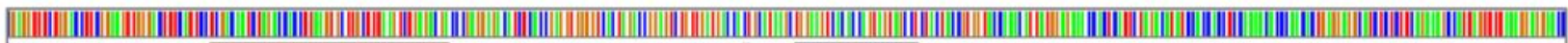

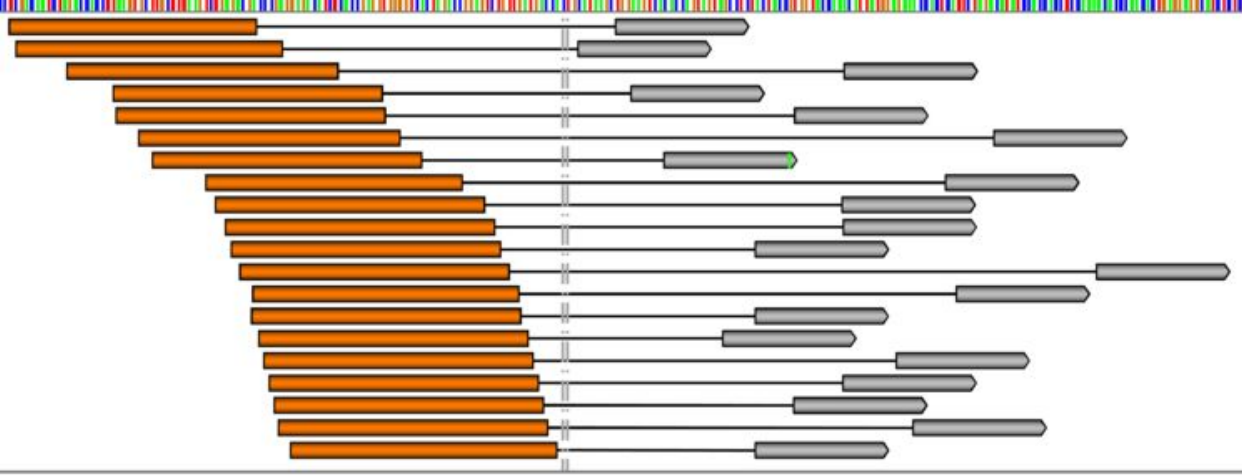

b

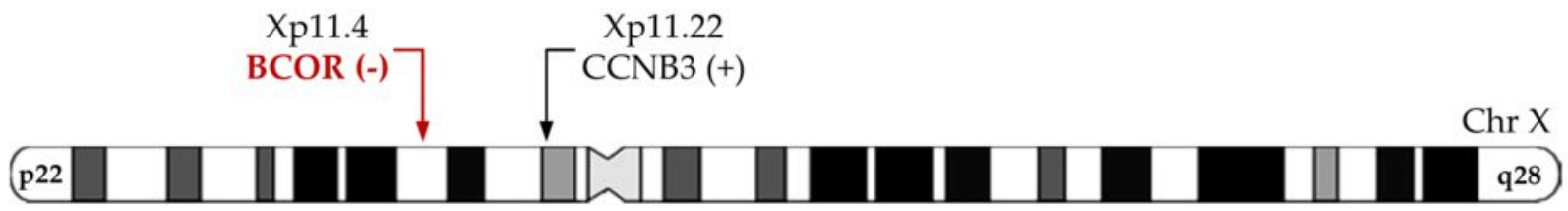

C

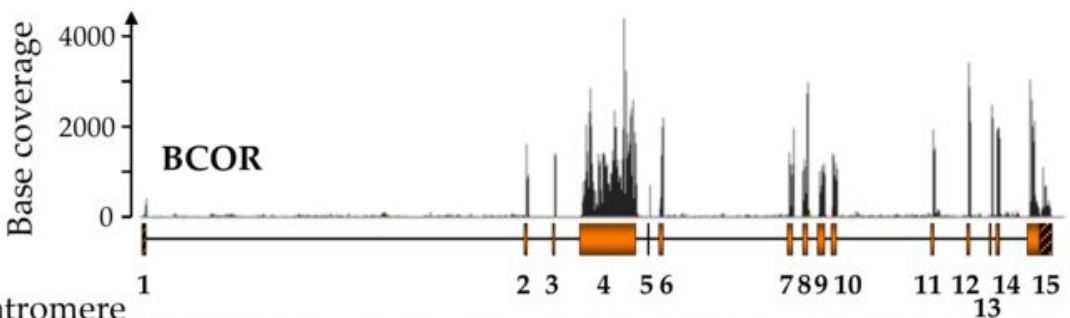

Centromere

$46.16 \mathrm{~Kb}$

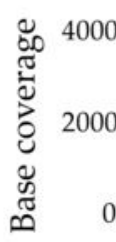

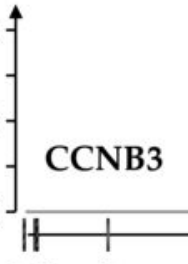

123

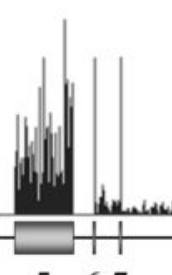

$5 \quad 67$

Telomere 1

作

\section{$67.37 \mathrm{~Kb}$}

d

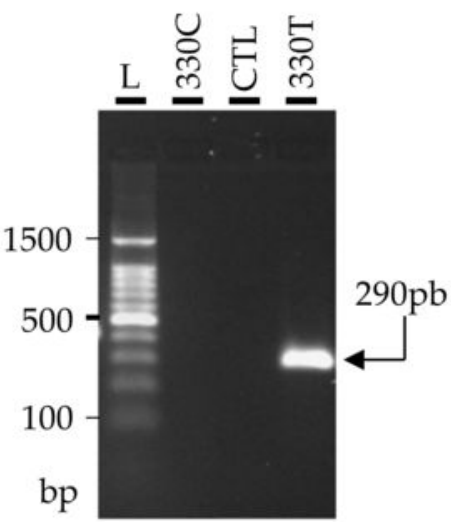

e

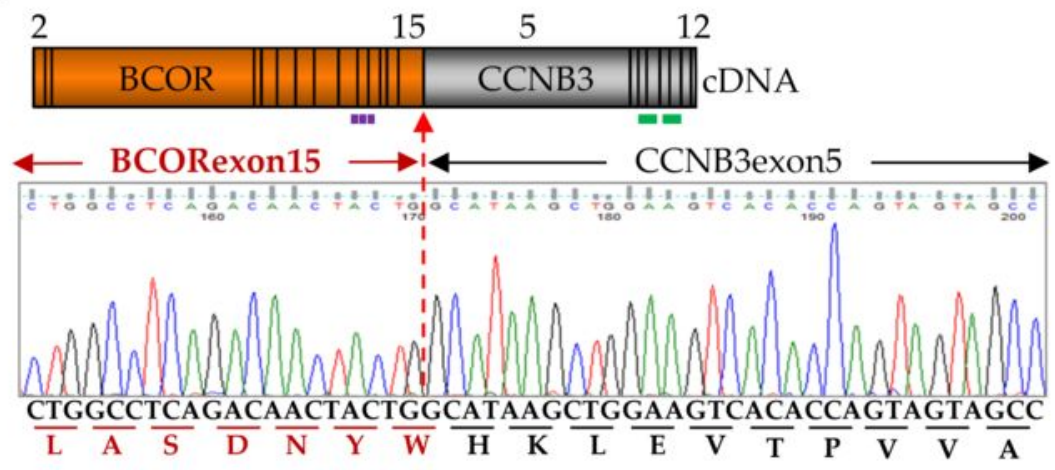


a
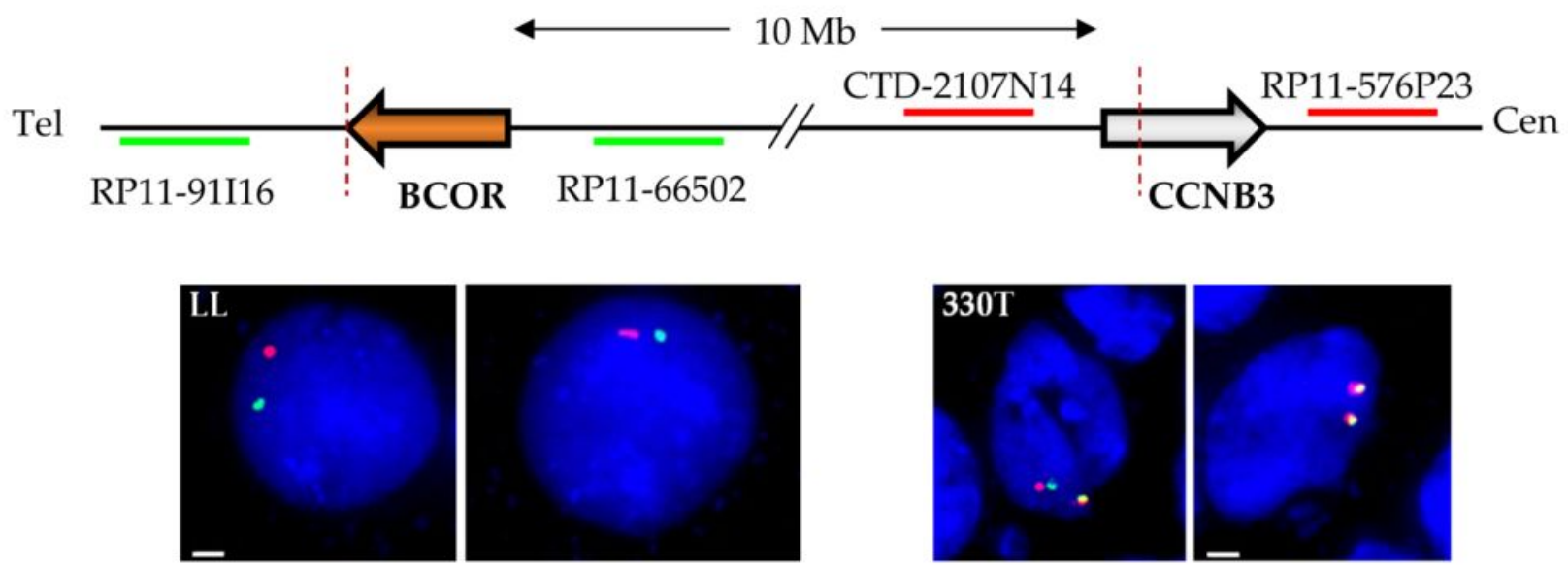

b
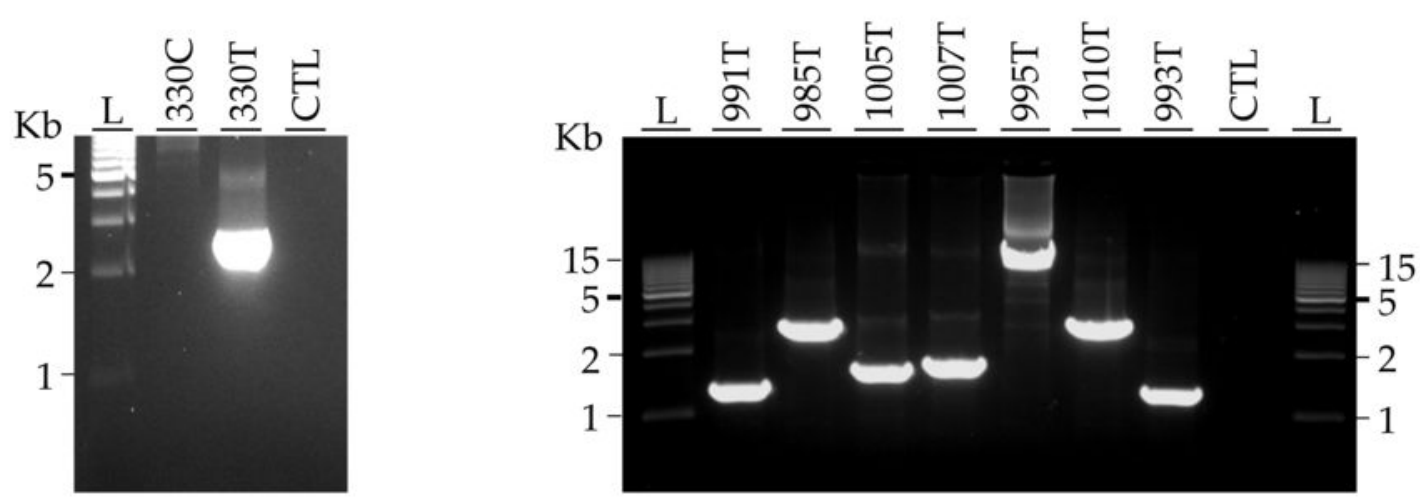

C

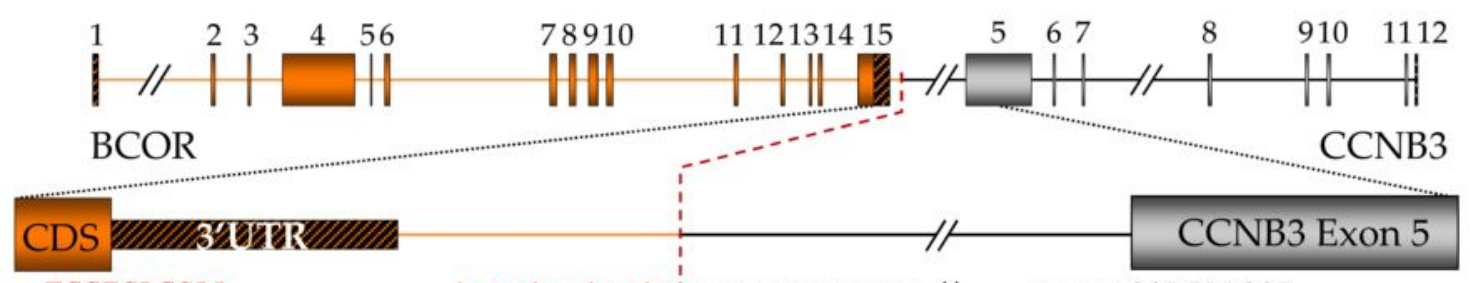

. TGGTGAGCAA. . . . . . . . . taaatggtgctgigtgtttttgagCa $/ f \ldots$. . cacagGCATAAGCT...

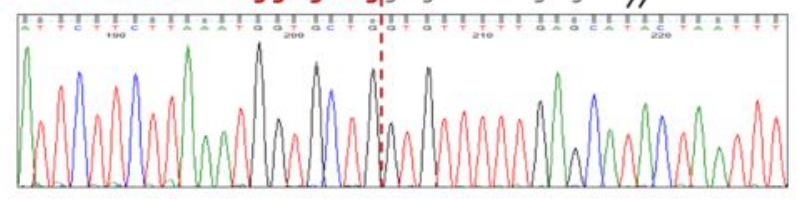

d

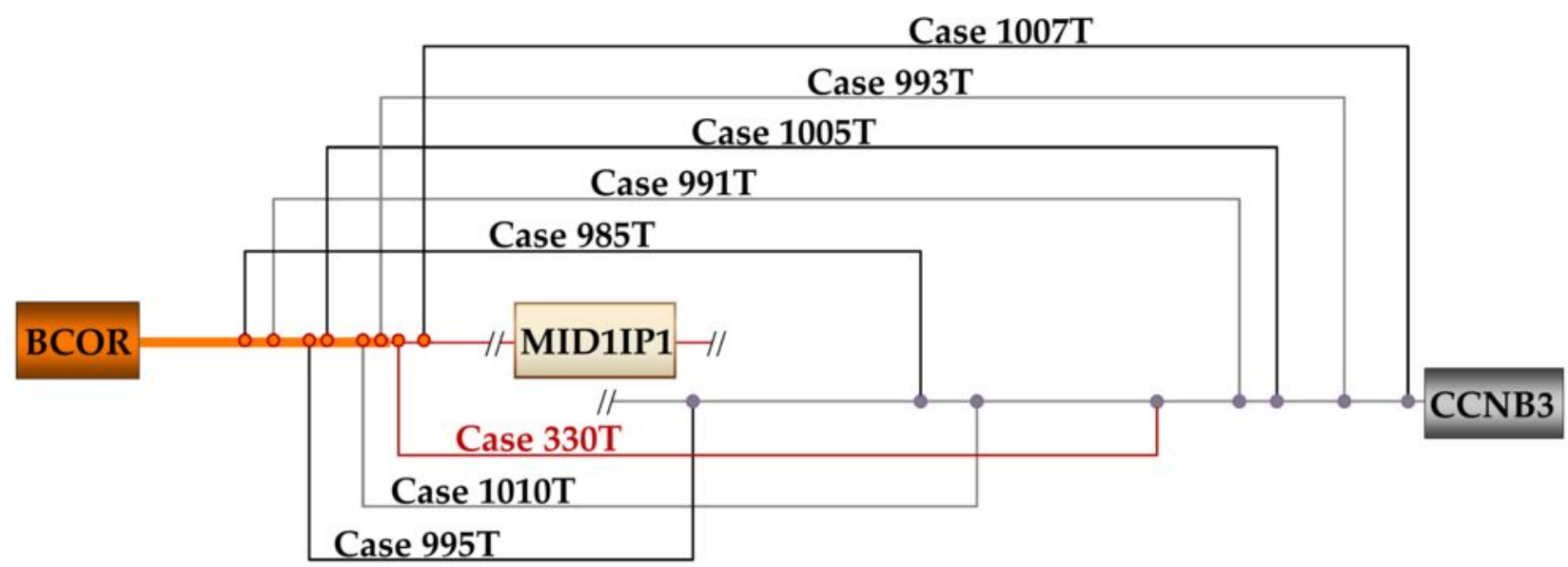




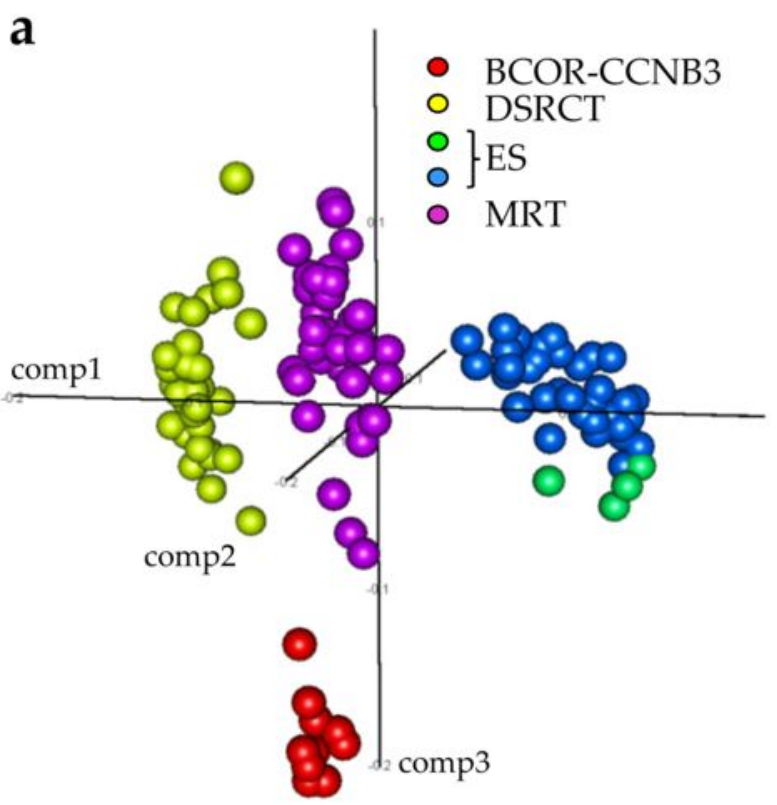

b

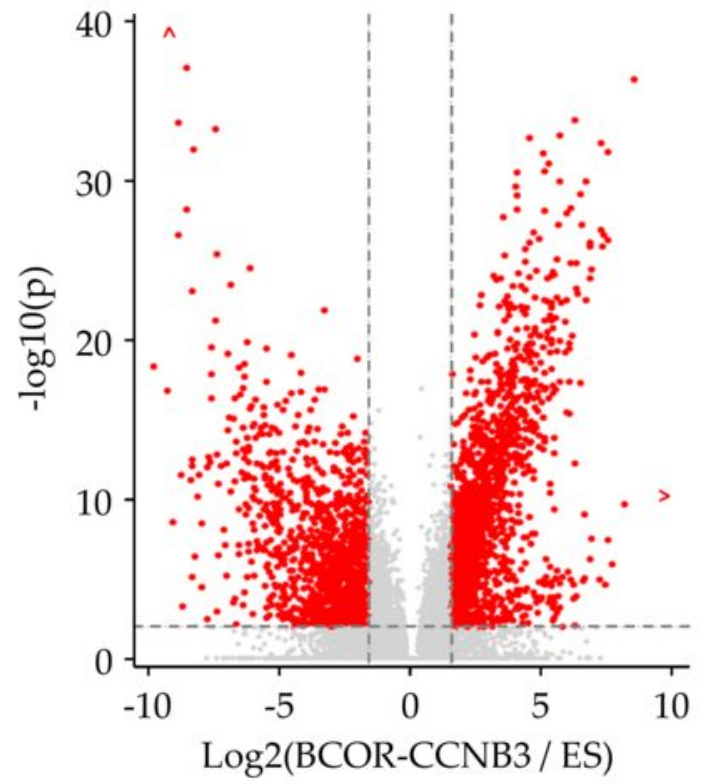

C

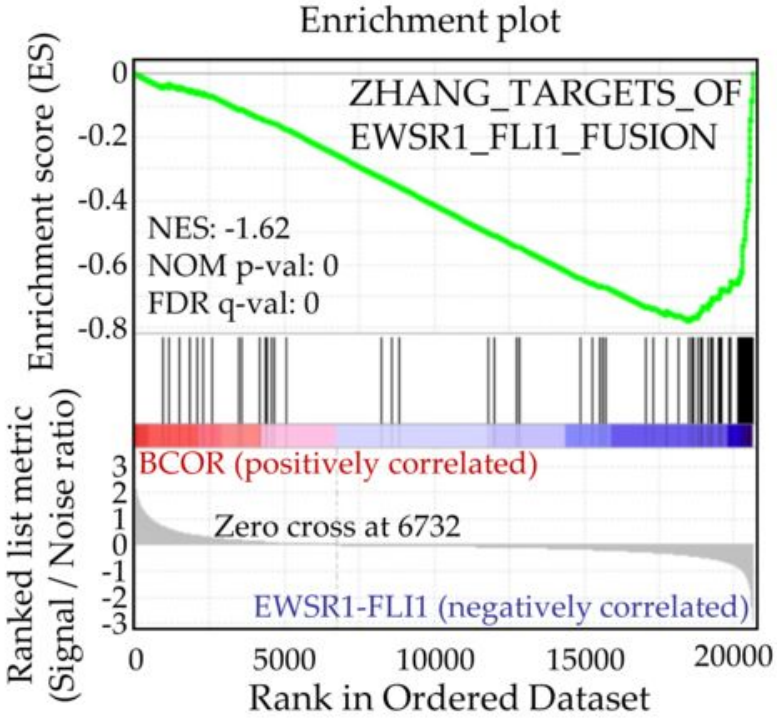

d

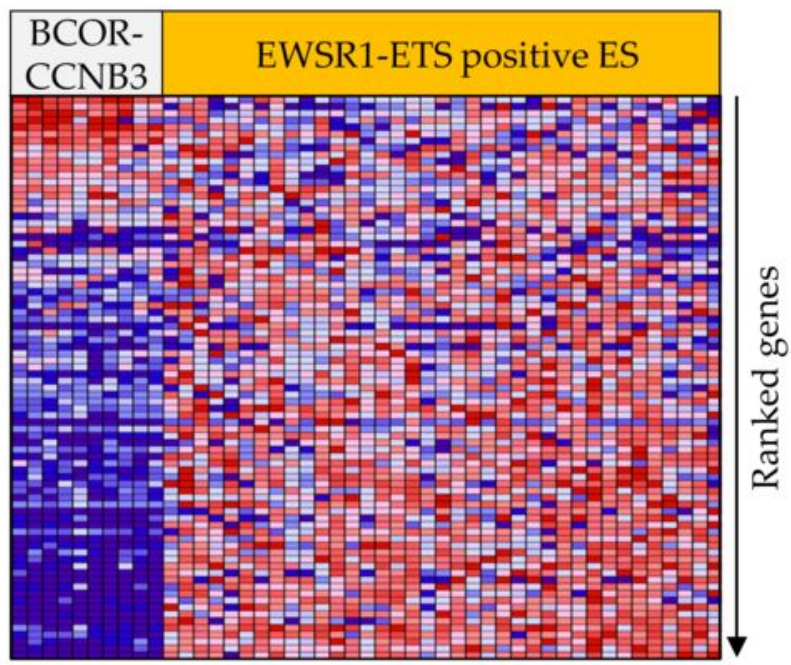


a

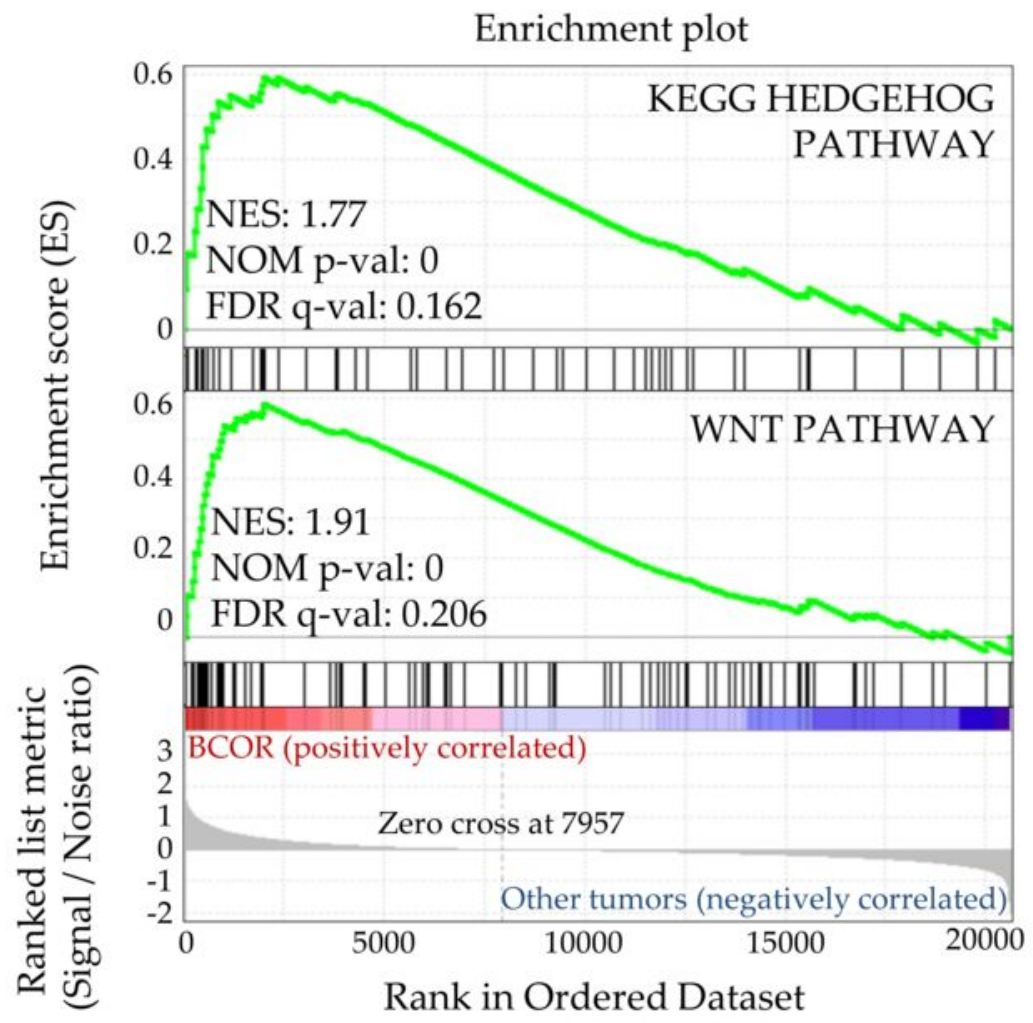

b

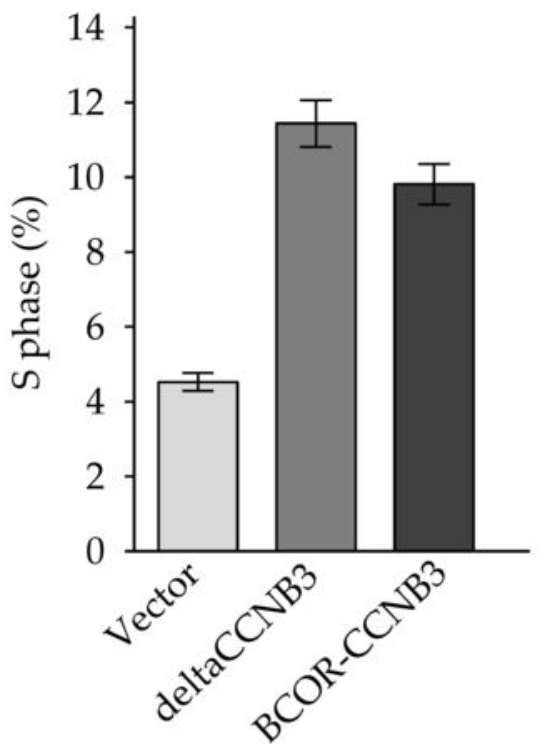


a

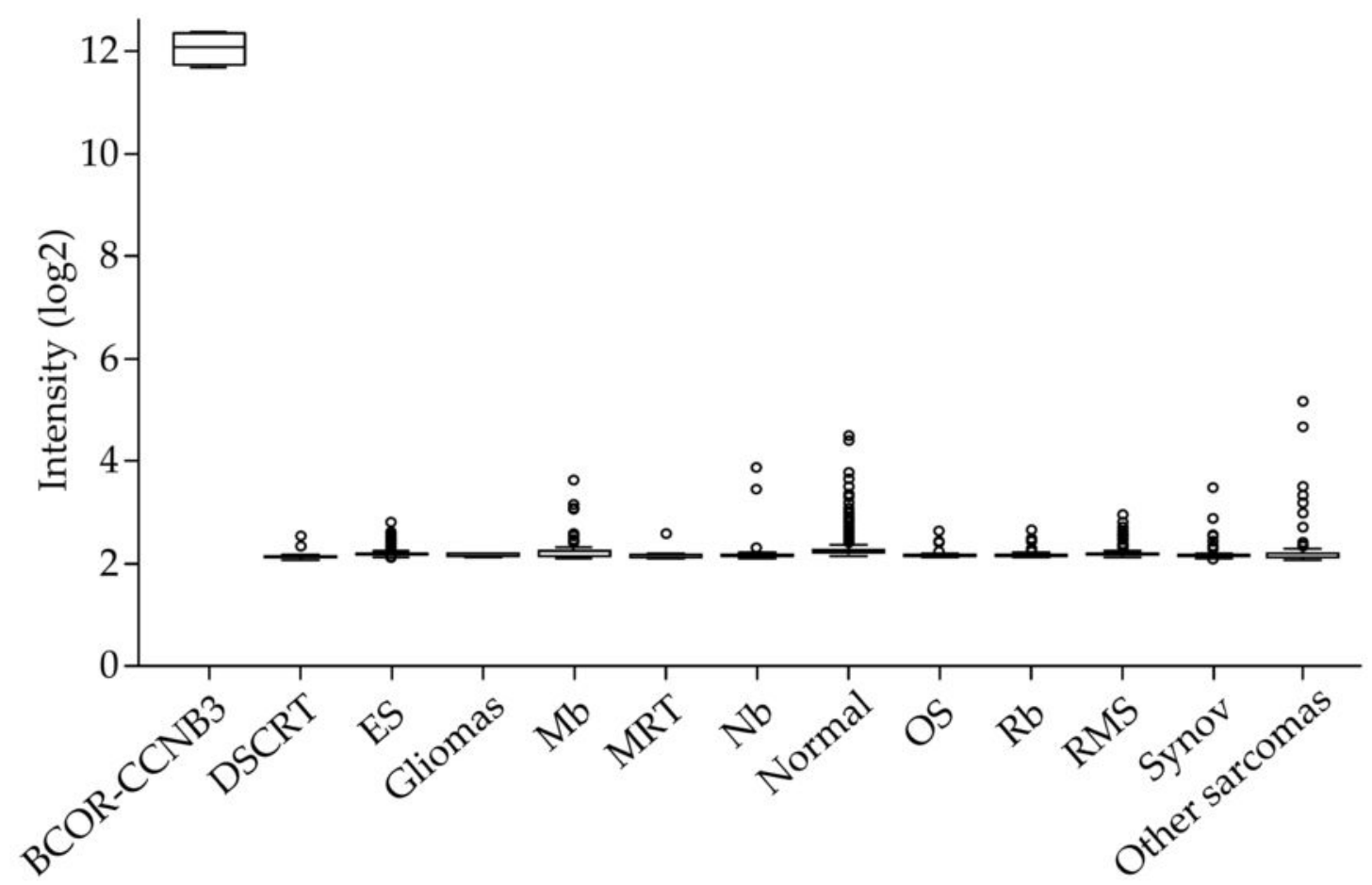

b

HES

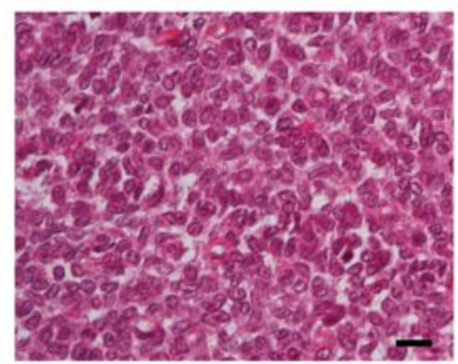

Ewing

BCOR-CCNB3 cases
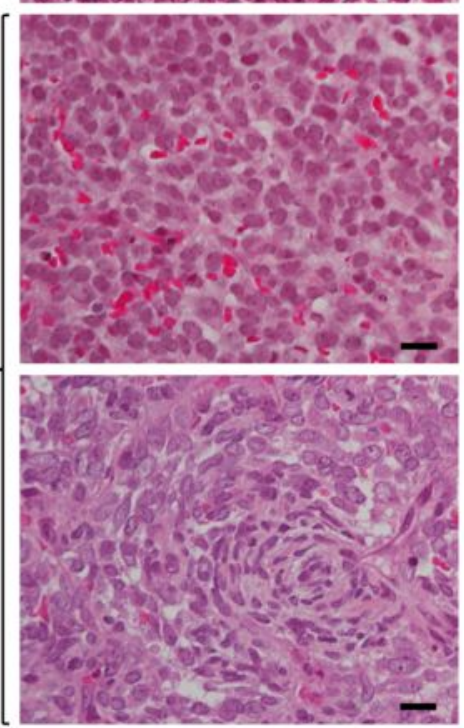

CD99
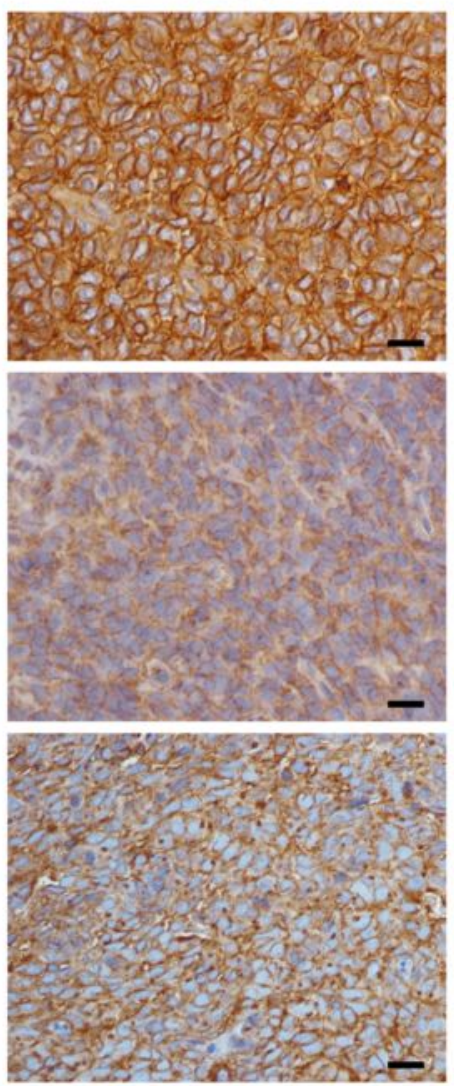

CCNB3
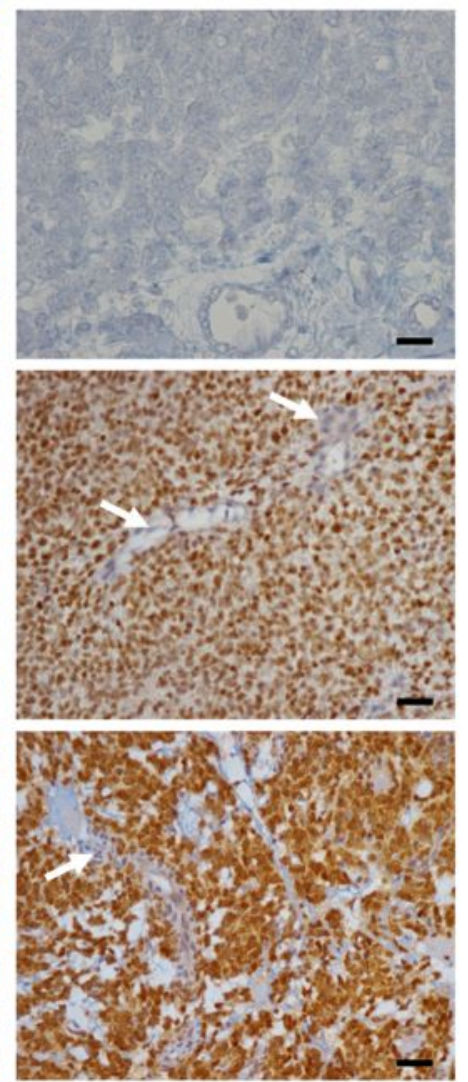


\section{A new subtype of bone sarcoma defined by BCOR-CCNB3 gene fusion}

Gaëlle PIERRON*, Franck TIRODE*, Carlo LUCCHESI*, Stéphanie REYNAUD, Stelly BALLET, Sarah COHEN-GOGO, Virginie PERRIN, Jean-Michel COINDRE and Olivier DELATTRE

\section{Supplementary Figure 1}

Decreased coverage at the stop codon position of BCOR in the 330T sample

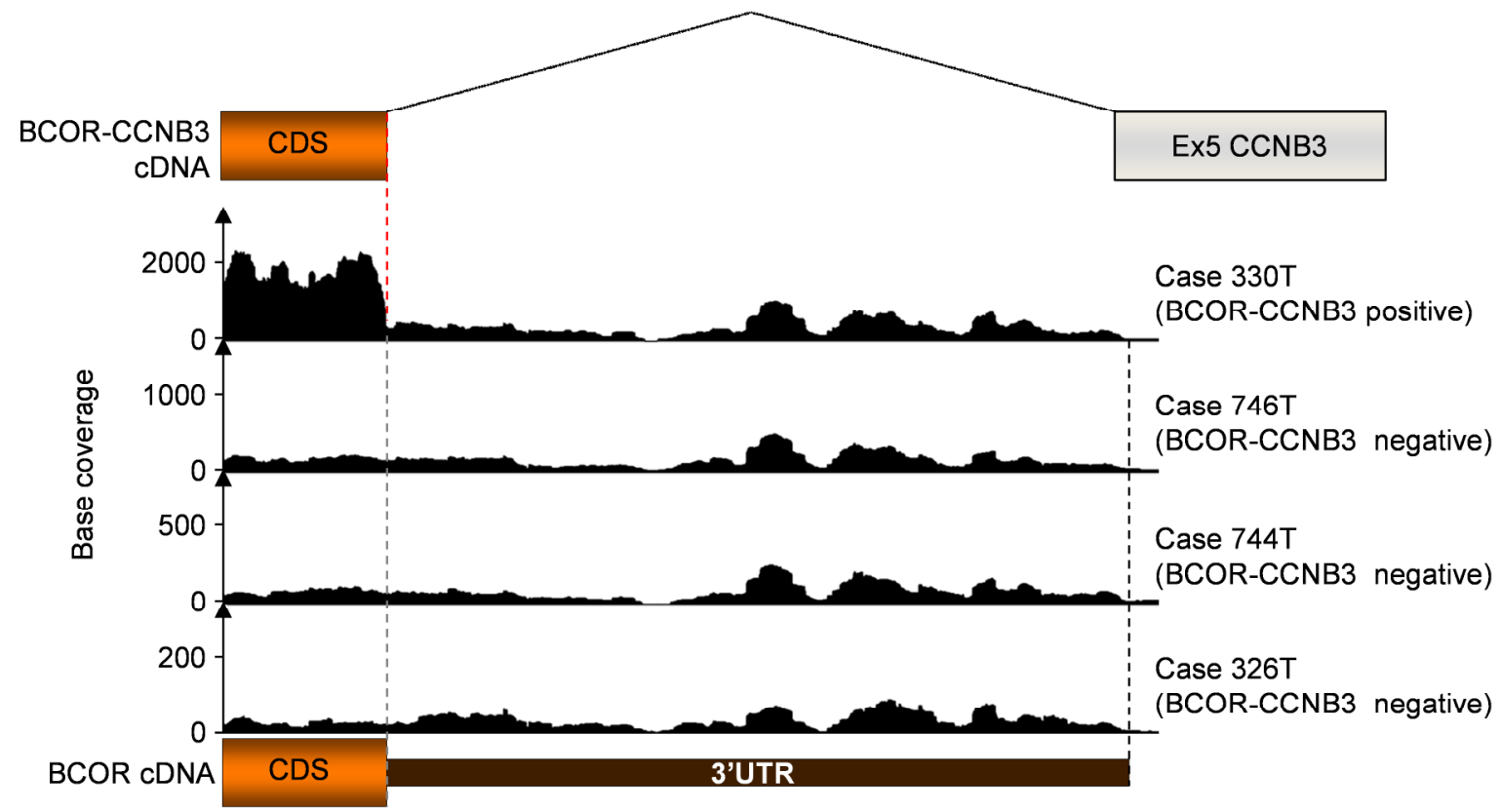

Zoom of the $3^{\prime}$ of the BCOR gene. The orange box indicates the coding sequence and the orange/black hatched line the 3' UTR. In case 330T a dramatic decrease of the number of reads mapping to the genomic BCOR sequence is observed at the end of the coding sequence (CDS) of exon 15. This decrease is not observed in the three other cases that were sequenced (grey line). Thus, in the 330T sample, the coding part of BCOR is expressed at higher level than the $3^{\prime} \mathrm{UTR}$ due to its fusion with CCNB3. Yet, there are still reads mapping to the BCOR 3' end which have to be generated from the same allele since this is a tumor from a male patient with a single chr $X$, as demonstrated by SNP 6.0 arrays. Hence, the presence of 3' BCOR UTR reads indicates that the wild type BCOR is also expressed in the 330T case as a result of incomplete usage of the crytic splice site provided by the BCOR STOP codon. This is further supported by microarray data that show that probesets covering the coding sequence of BCOR are expressed at higher levels in BCOR-CCNB3-positive cases as compared to other tumor types whereas probesets covering the $3^{\prime}$ BCOR UTR demonstrate an expression level similar to other tumors (supplementary figure 2). 


\section{Supplementary Figure 2}

Expression of BCOR across a variety of normal tissues and cancers
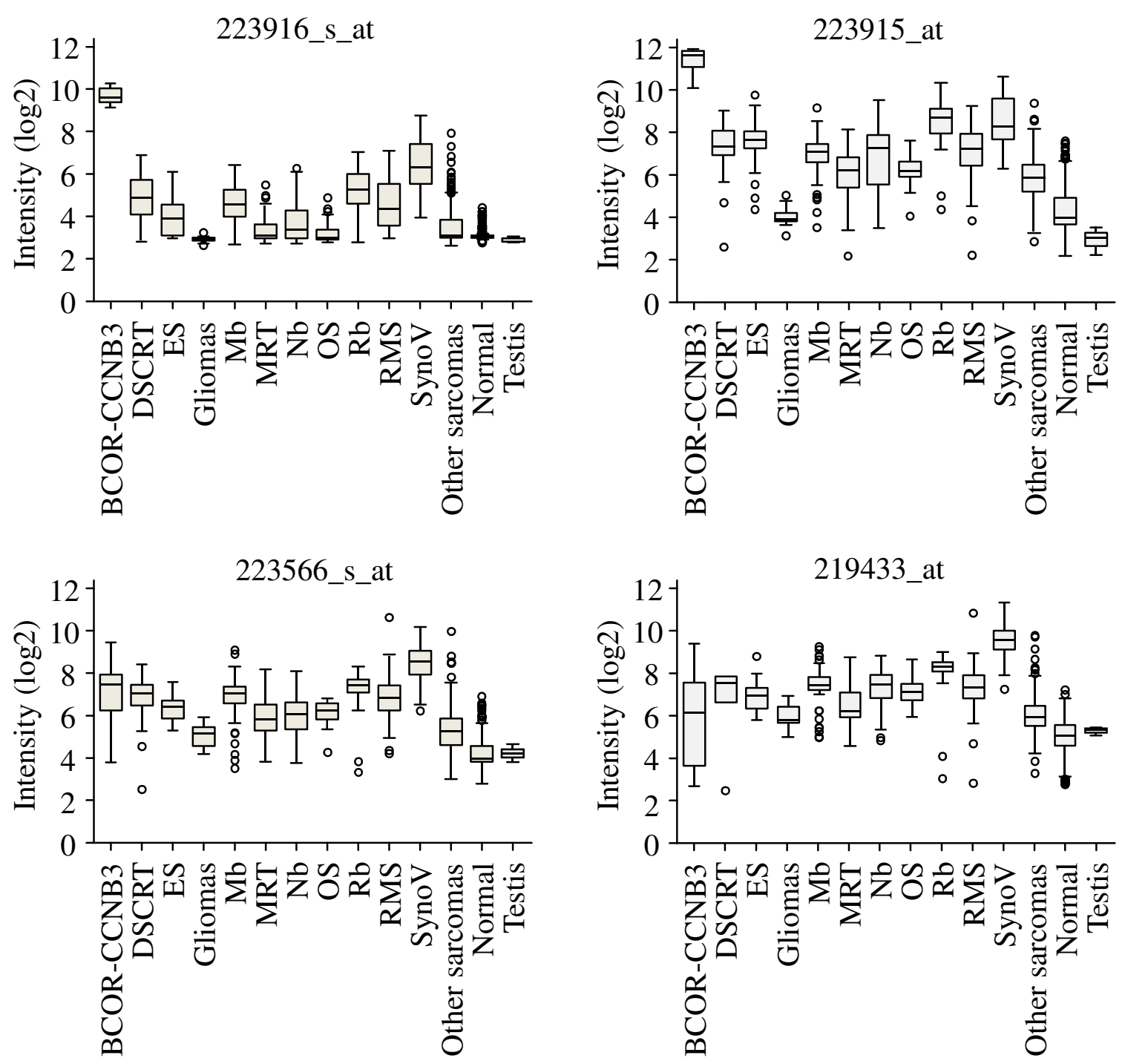

The BCOR gene is covered by four distinct probesets. Two of them, 223916_s_at and 223915_at include probes located along the coding sequence, and demonstrate a higher signal in BCOR-CCNB3-positive cases than in other tumors or normal tissues. These probesets interrogate both the wild-type BCOR and the BCOR-CCNB3 fusion. In contrast, 219433_at and 223566_s_at probesets are located within the BCOR 3' UTR. They indicate a signal similar in BCOR-CCNB3-positive cases and in other tumors or normal tissues. Only the wild type BCOR is evaluated by these probesets. 


\section{Supplementary Figure 3}

Heat map of the genes included in the Zhang_Targets_of_EWSR1_FLI1 fusion signature

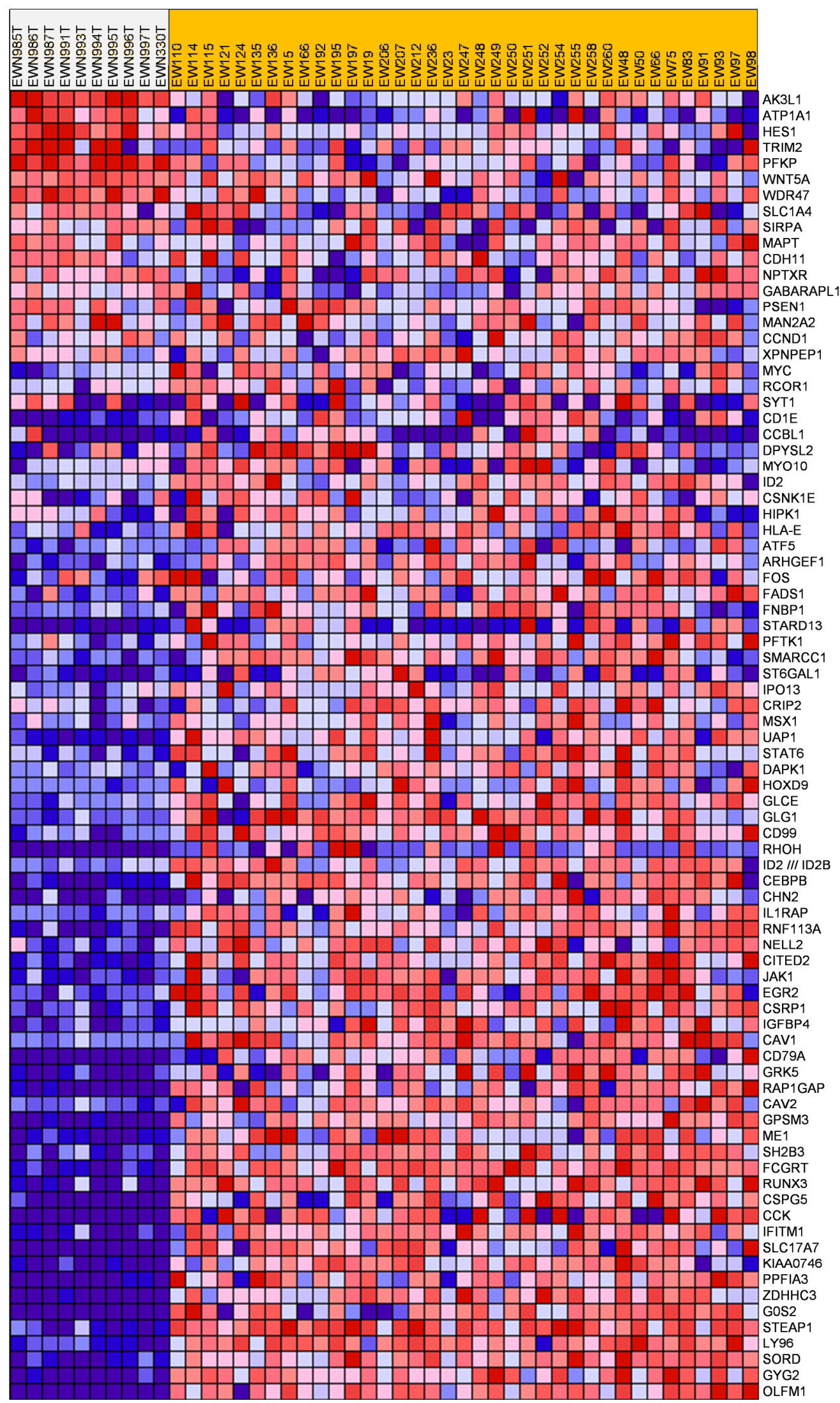




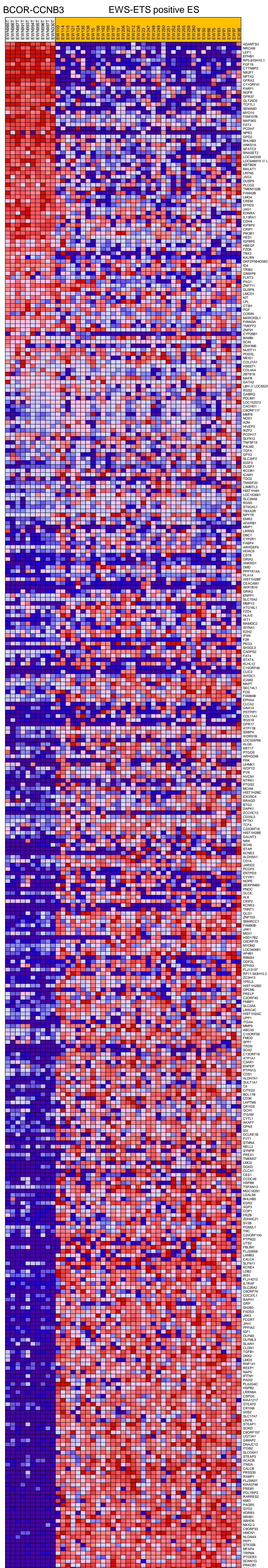




\section{Supplementary Figure 5: Complete nucleotide and peptide sequences of BCOR- CCNB3}

\section{Homo sapiens BCL6 corepressor - cyclin B3 (BCOR-CCNB3), CDS}

1 atgctctcagcaacccccctgtatgggaacgttcacagctggatgaacagcgagagggtc

61 cgcatgtgtggggcgagcgaagacaggaaatccttgtaaatgatggtgacgcttcaaaa

121 gccagactggaactgagggaagagaatcccttgaaccacaacgtggtggatgcgagcacg

181 gcccataggatcgatggcctggcagcactgagcatggaccgcactggcctgatccgggaa

241 gggctgcgggtcccgggaacatcgtctattctagcttgtgtggactgggctcagagaaa

301 ggtcgggaggctgccacaagcactctaggtggccttgggttttcttcggaaagaaatcca

361 gagatgcagttcaaaccgaatacacccgagacagtggaggcttctgccgtctctggaaaa

421 cccccaatggcttcagtgctatatacaaacaccgcctggaatacaaaaagtgctgta

481 gccacagcagaagcgctgggcttggacaggcctgccagcgacaaacagagccctctcaac

541 atcaatggtgctagttatctgcggctgccctgggtcaatccttacatggagggtgccacg

601 ccagccatctacccttcctcgactcgccaataagtattcactgaacatgtacaaggcc

661 ttgctacctcagcagtcctacagcttggcccagccgctgtattctccagtctgcaccaat

721 ggggagcgctttctctacctgccgccacctcactacgtcggtccccacatcccatcgtcc

781 ttggcatcacccatgaggctctcgacaccttcggcctccccagccatcccgcctctcgtc

841 cattgcgcagacaaagectcccgtggaagatgggcgtcagccetgggaatcctgttgat

901 tcccacgcctatcctcacatccagaacagtaagcagcccagggttccctctgccaaggcg

961 gtcaccagtggcctgccgggggacacagctctcctgttgcccccctcgcctcggccgtca

1021 ccccgagtccaccttcccacccagcctgctgcagacacctactcggagttccacaagcac

1081 tatgccaggatctccacctctccttcagttgccctgtcaaagccatacatgacagttagc

1141 agcgagttccccgcggccaggctctccaatggcaagtatcccaaggctccggaagggggc

1201 gaaggtgcccagccagtgcccgggcatgcccggaagacagcggttcaagacagaaaagat

1261 ggcagctcacctcctctgttggagaagcagaccgttaccaaagacgtcacagataagcca

1321 ctagacttgtcttctaaagtggtggatgtagatgcttccaaagctgaccacatgaaaag

1381 atggctcccacggtcctggttcacagcagggctggaagtggcttagtgctctccggaagt

1441 gagattccgaaagaacactatctcctccaggaaatggttgtgctatctatagatctgaa

1501 atcatcagcactgctccctcatcctgggtggtgcccgggccaagtcctaacgaagagaac

1561 aatggcaaaagcatgtcgctgaaaacaaggcattggactgggcgataccacagcagcgg

1621 agttcatcatgcccgcgcatgggcggcaccgatgctgtcatcactaacgtttcagggtca

1681 gtgtcgagtgcaggccgcccagcctccgcatcacccgcccccaltgccaatgcagatggc

1741 accaaaccagcaggagctctgtagaaaccacaccatccgttattcagcacgtgggccag

1801 cccccggccactcctgccaagcacagtagcagcaccagcagcaagggcgccaaagccagc

1861 aacccagaaccgagtttcaaagcaaacgagaacggccttccaccaagctctatatttctg

1921 tctccaaatgaggcattcaggtccccaccaattccctaccccaggagttacctcccttac

1981 ccagcccctgagggcattgctgtaagtcccctctccttacatggcaaaggacctgtctac

2041 cctcacccagttttgttacccaatggcagtctgtttcctgggcaccttgccccaagcct

2101 gggctgccctatgggcttcccaccggccgtccagagtttgtgacctaccaagatgccctg

2161 gggttgggcatggtgcatcccatgttgataccacacacgcccatagagattactaaagag

2221 gagaaaccagagaggagatcccggtcccatgagagagcccgttacgaggacccaaccctc

2281 cggaatcggttttccgagattttggaaactagcagcaccaagttacatccagatgtcccc

2341 accgacaagaacctaaagccgaaccccaactggaatcaagggaagactgttgtcaaagc

2401 gacaagcttgtctacgtagaccttctccgagaagaaccagatgctaaaactgacacaaac

2461 gtgtccaaacccagctttgcagcagagagtgttggccagagcgctgagccccccaagccc

2521 tcagttgagccggccctgcagcagcaccgtgatttcatcgccctgagagaggagttgggg

2581 cgcatcagtgacttccacgaaacttatactttcaaacagccagtcttcaccgtaagcaag

2641 gacagtgttctggcaggtaccaacaaagagaacctagggttgccagtctcgactccattc

2701 ctggagccacctctggggagcgatggccetgctgtaacttttggtaaaaccaagaggat

2761 cccaaaccattttgtgtgggcagtgccccaccaagtgtggatgtgacccccacctatacc

2821 aaagatggagctgatgaggctgaatcaaatgatggcaaagttctgaaaccgaagccatct

2881 aagctggcaaagagaatcgccaactcagcgggttacgtgggtgaccgattcaatgtgtc

2941 actaccgaactgtatgcagattccagtcagctcagccgggagcaacgggcattgcagatg

3001 gaaggattacaagaggacagtattttatgtctacccgctgcttactgtgagcgtgcaatg

3061 atgcgcttctcagagttggagatgaaagaaagagaaggtggccacccagcaaccaaagac

3121 tccgagatgtgcaaattcagcccagccgactgggaaaggttgaaaggaaatcaggacaaa 
3181 aagccaaagtcggtcaccctggaggaggccattgcagaacagaacgaaagtgagagatgc 3241 gagtatagtgttggaaacaagcaccgtgatccctttgaagccccagaggacaaagatctt 3301 cctgtggagaagtactttgtggagaggcagcctgtgagcgagcctcccgcagaccaggtg 3361 gcctcggacatgcctcacagccccaccctccgggtggacaggaaacgcaaagtctcaggt 3421 gacagcagccacactgagaccactgcggaggaggtgccagaggaccctctgctgaaagcc 3481 aarcgccgacgagtctctaaagatgactggcctgagagggaaatgacaaacagttcctct 3541 aaccacttagaagacccacattatagtgagctgaccaacctgaaggtgtgcattgaatta 3601 acagggctccatcctaaaaacaacgccacttgctgcaccttagagaacgatgggagcag 3661 caggtgtcggcagcagatggcaaacctggccggcaaagcaggaaggaagtgacccaggcc 3721 actcagcctgaggccattcctcaggggactaacatcactgaagagaaacctggcaggaaa 3781 agggcagaggccaaaggcaacagaagctggtcggaagagtctcttaaacccagtgacaat 3841 gaacaaggcttgcctgtgttctccggctctccgcccatgaagagtctttcatccaccagt 3901 gcaggcggcaaaagcaggctcagccaagctgcgcaccagcctccaggccgcctgccaaa 3961 cagcagaaattaaagaaaccagaagacagatgtgctgtgtgcagacgaagaagaggat 4021 tgccaggctgcctccctgctgcagaaatacaccgacaacagcgagaagccatccgggaag 4081 agactgtgcaaaccaaacacttgatccctcaggagtccaggcggggattgccactgaca 4141 ggggaatactacgtggagaatgccgatggcaaggtgactgtccggagattcagaaagcgg 4201 ccggagcccagttcggactatgatctgtcaccagccaagcaggagccaaagcccttcgac 4261 cgcttgcagcaactgctaccagcctcccagtccacacagctgccatgctcaagttcccct 4321 caggagaccacccagtctcgccctatgccgccggaagcacggagacttattgtcaataag 4381 aacgctggcgagacccttctgcagcgggcagccaggcttggctatgaggaagtggtcctg 4441 tactgcttagagaacaagatttgtgatgtaatcatcgggacaacgcaggttactgcgcc 4501 ctgcatgaagcttgtgctaggggctggctcaacattgtgcgacacctccttgaatatggc 4561 gctgatgtcaactgtagtgcccaggatggaaccaggcctctgcacgatgctgttgagaac 4621 gatcacttggaaattgtccgactacttctctcttatggtgctgaccccaccttggctacg 4681 tactcaggtagaaccatcatgaaatgacccacagtgaacttatggaaaagttcttaaca 4741 gattatttaatgacctccagggtcgcaatgatgatgacgccagtggcacttgggacttc 4801 tatggcagctctgtttgtgaaccagatgatgaaagtggctatgatgttttagccaacccc 4861 ccaggaccagaagaccaggatgatgatgacgatgcctatagcgatgtgtttgaatttgaa 4921 ttttcagagacccccctcttaccgtgttataacatccaagtatctgtggctcaggggcca 4981 cgaaactggctactgctttcggatgtccttaagaaattgaaatgtcctcccgcatattt 5041 cgctgcaattttccaaacgtggaaattgtcaccattgcagaggcagaattttatcggcag 5101 gtttctgcaagtctcttgttctcttgctccaaagacctggaagccttcaaccctgaaagt 5161 aaggagctgttagatctggtggaattcacgaacgaaattcagactctgctgggctcctct 5221 gtagagtggctccaccccagtgatctggcctcagacaactactggcataagctggaagtc 5281 acaccagtagtagcctctactaccgtggtaccaaacattatggagaaaccactcattcta 5341 gacatatccaccacctccaaacacccaacactgaggaggcatctctcttcagaagcca 5401 ttagttttaaaggaggaacccactattgaggatgaaacccttatcaataagtcattatct 5461 ttaaaaagtgctcaaatcatgaggaggtgtccttactggaaaagctacagcccctgcag 5521 gaggagagtgacagtgatgatgcgtttgttatagagccaatgacttttaagaagacacat 5581 aaactgaggaggcagccatcaccaagaagacattatccttaagaagaagatgtgtgca 5641 agtcagcggaagcagtcctgccaggaagagtcgttggctgtgcaggatgtcaatatggaa 5701 gaggattccttctttatggagtcaatgagttttaagaagaagcctaaaactgaggagtca 5761 atccccacccataagttatcatctttaaagaagaatgtaccatttatgggaagatatgc 5821 cactttaggaagccaccagtattgcagacaaccatctgtggagcaatgtcctccattaag 5881 aagcctaccactgagaaggagacactttccaagagctatctgtattgcaagagaaacac 5941 accactgagcatgagatgtccatcttgaagaatcattggccttgcagaagaccaacttt 6001 aagaggattcccttgttaaggagtcgttagcctttaagaagaagcctagcactgaggag 6061 gcaatcatgatgccagtaatattgaaggagcagtgcatgactgaggggaagaggtcccgt 6121 ctgaagccattagtattgcaggagatcacctctggagagaagtcgctcattatgaagcca 6181 ttgtccattaaagaaagccatctactgagaaggagtcctttcccaggaaccatctgca 6241 ttgcaaaagaagcacaccactcaggaggaggtttccatcttaaaggagccctcgtccttg 6301 ctaagtctccaactgaggagtcaccttttgatgaggctttggcttttacaaagagtgt 6361 accattgaggaggcaccccccaccaagaagcctttaattttaaagaggaagcatgccact 6421 caggggacaatgtcccacttgaagaaaccactaatattacagaccacctctggagaaag 6481 tcacttattaaggagccactgccctttaagaagaaaagtgtctttaaagaaaagtgt 6541 accacacaagagatgatgtccatctgtccagaactgttggactttcaggatatgattggt 6601 gaagataagaattctttctttatggagccaatgtcatttaggaagaaccctacaactgag 6661 gagacagtacttaccaagacatcgttgtctttacaggaaaagaaattactcaggggaag 6721 atgtcccacttaagaagccactggtcttgcagaagatcacttctgaggaggagtcattc 6781 tataagaagctgttgccctttaagatgaaatctacaacggaagaaaagttcctctcccag 
6841 gaaccatctgcattgaaagagaagcataccaccttgcaggaagtgtccctctcaaagag 6901 tcattggccatccaagagaaggctaccactgaggaggaattctctcaggaactattttca 6961 ttgcatgttaagcataccaacaaagtgggtccctcttccaggaggctttggtcttgcaa 7021 gagaagactgatgccgaagaggattccttgaagaacttgttggctttgcaggagaaagc 7081 accatggaagaagagtcccttatcaataagctattggctctgaaggaggagctttctgct 7141 gaggcagccacaaacatacagacacaattatctttaagaagaagtccacttctcatgga 7201 aagtgttcttcctgaagaagcagttggctttgaatgagaccatcaatgaagaggagttc 7261 cttaataagcagccactggccttggaggggtatcccagcattgcggagggggagaccctc 7321 ttcaagaagcttttggccatgcaggaggagcccagcattgagaaggaagctgtcctcaag 7381 gagcccactattgacacagaagctcactttaaggaacctttggccttgcaggaggagccc 7441 agcactgagaaggaggctgtcctcaaggagcccagtgttgacacagaagctcactttaag 7501 gaaactttggccttgcaggagaagcccagcattgagcaggaggccctctttaagcgacac 7561 tcagctttgtgggagaagcccagcactgagaaggagaccatcttcaaggagtctttggac 7621 ttgcaagagaagcccagcattaagaaagagaccctcctcaaaagccattagccttgaag

7681 atgtctaccatcaatgaggcagtcctcttcgaagatatgatagctctgaatgagaaaccc

7741 accactgggaaggagttgtccttcaaggagccattagccttacaagagagtcccacctac

7801 aaggaagacacctttctcaaacattgttggtcccccaagttggaaccagcccaatgtg

7861 tctagcactgcccctgaatccataaccagcaagtccagcattgctaccatgaccagtgtg 7921 ggcaalctggtaccatcaatgaggcattcctcttcgaagatatgataactctgaatgag 7981 aaacccaccactgggaaggagttgtccttcaaggagccattggccttacaagagagtccc 8041 acctgcaaggaagacacctttctggaaacattcttgatcccccaaattggaaccagccca 8101 tatgtgtttagcaccacccctgaatccataacagagaagtccagcattgcaaccatgacc 8161 agcgtgggcaagtccaggaccaccaccgagtccagtgcatgtgaatctgcttctgataaa 8221 cctgtctcaccacaggccaagggaacaccaaaggagataaccccacgggaagatattgat 8281 gaggacagcagtgatccaagtttcaacccaatgtatgccaaggaaatcttcagttacatg 8341 aagagagagaggaacagtttatacttacagattacatgaacaggcagattgaatcacc 8401 agtgacatgagggccattcttgtggactggttggtggaggtgcaggtgtcctttgagatg 8461 acccatgagaccctgtacttggcagtgaagctggtggatctctacctaatgaaggcagta 8521 tgcaagaaggataagttacaactccttggtgccactgcctttatgattgcagcaaattt 8581 gaggagcacaactcacctcgtgtggatgactttgtgtacatctgtgatgataattatcag 8641 cgatctgaggtactcagcatggaatcaacatcctgaacgtcctcaatgtgacattaac 8701 attcccatcgcctaccattttctgcgcagatatgctaggtgtatccacaccaacatgaag 8761 acactgaccttgtcccgctacatctgcgagatgaccctgcaggaataccactatgtccag 8821 gagaaggcttccaagctagctgctgcctccttactcctggccctctacatgaagaagctc 8881 ggatactgggttcccttcctggagcattacagtggctacagtatctctgagcttcacccc 8941 ttggtcagacagctgaacaaactgctgactttcagttcttacgatagtctcaaggctgtg 9001 tattacaagtattctcacccggtcttctttgaagtcgccaaatccctgccttggatatg 9061 ttgaagctggaggagattttgaactgtgattgtgaggctcagggcctggtactctag

\section{BCOR-CCNB3 amino acid sequence}

1 MLSATPLYGNVHSWMNSERVRMCGASEDRKILVNDGDASKARLELREENPLNHNVVDAST

61 AHRIDGLAALSMDRTGLIREGLRVPGNIVYSSLCGLGSEKGREAATSTLGGLGFSSERNP

121 EMQFKPNTPETVEASAVSGKPPNGFSAIYKTPPGIQKSAVATAEALGLDRPASDKQSPLN

181 INGASYLRLPWVNPYMEGATPAIYPFLDSPNKYSLNMYKALLPQQSYSLAQPLYSPVCTN

241 GERFLYLPPPHYVGPHIPSSLASPMRLSTPSASPAIPPLVHCADKSLPWKMGVSPGNPVD

301 SHAYPHIQNSKQPRVPSAKAVTSGLPGDTALLLPPSPRPSPRVHLPTQPAADTYSEFHKH

361 YARISTSPSVALSKPYMTVSSEFPAARLSNGKYPKAPEGGEGAQPVPGHARKTAVQDRKD

421 GSSPPLLEKQTVTKDVTDKPLDLSSKVVDVDASKADHMKKMAPTVLVHSRAGSGLVLSGS

481 EIPKETLSPPGNGCAIYRSEIISTAPSSWVVPGPS PNEENNGKSMSLKNKALDWAIPQQR

541 SSSCPRMGGTDAVITNVSGSVSSAGRPASASPAPNANADGTKTSRSSVETTPSVIQHVGQ

601 PPATPAKHSSSTSSKGAKASNPEPSFKANENGLPPSS IFLSPNEAFRSPPIPYPRSYLPY

661 PAPEGIAVSPLSLHGKGPVYPHPVLLPNGSLFPGHLAPKPGLPYGLPTGRPEFVTYQDAL

721 GLGMVHPMLIPHTPIEITKEEKPERRSRSHERARYEDPTLRNRFSEILETSSTKLHPDVP

781 TDKNLKPNPNWNQGKTVVKSDKLVYVDLLREEPDAKTDTNVSKPSFAAESVGQSAEPPKP

841 SVEPALQQHRDFIALREELGRISDFHETYTFKQPVFTVSKDSVLAGTNKENLGLPVSTPF

901 LEPPLGSDGPAVTFGKTQEDPKPFCVGSAPPSVDVTPTYTKDGADEAESNDGKVLKPKPS

961 KLAKRIANSAGYVGDRFKCVTTELYADSSQLSREQRALQMEGLQEDSILCLPAAYCERAM

1021 MRFSELEMKEREGGHPATKDSEMCKFSPADWERLKGNQDKKPKSVTLEEAIAEQNESERC

1081 EYSVGNKHRDPFEAPEDKDLPVEKYFVERQPVSEPPADQVASDMPHSPTLRVDRKRKVSG 
1141 DSSHTETTAEEVPEDPLLKAKRRRVSKDDWPEREMTNSSSNHLEDPHYSELTNLKVCIEL

1201 TGLHPKKQRHLLHLRERWEQQVSAADGKPGRQSRKEVTQATQPEAI PQGTNITEEKPGRK

1261 RAEAKGNRSWSEESLKPS DNEQGLPVFSGSPPMKSLSSTSAGGKKQAQPSCAPASRPPAK

1321 QQKIKENQKTDVLCADEEEDCQAASLLQKYTDNSEKPSGKRLCKTKHLIPQESRRGLPLT

1381 GEYYVENADGKVTVRRFRKRPEPSSDYDLSPAKQEPKPFDRLQQLLPASQSTQLPCSSSP

1441 QETTQSRPMPPEARRLIVNKNAGETLLQRAARLGYEEVVLYCLENKICDVNHRDNAGYCA

1501 LHEACARGWLNIVRHLLEYGADVNCSAQDGTRPLHDAVENDHLEIVRLLLSYGADPTLAT

1561 YSGRTIMKMTHSELMEKFLTDYLNDLQGRNDDDASGTWDFYGSSVCEPDDESGYDVLANP

1621 PGPEDQDDDDDAYSDVFEFEFSETPLLPCYNIQVSVAQGPRNWLLLSDVLKKLKMSSRIF

1681 RCNFPNVEIVTIAEAEFYRQVSASLLFSCSKDLEAFNPESKELLDLVEFTNEIQTLLGSS

1741 VEWLHPSDLASDNYWHKLEVTPVVASTTVVPNIMEKPLILDISTTSKTPNTEEASLERKP

1801 LVLKEEPTIEDETLINKS LSLKKCSNHEEVSLLEKLQPLQEESDSDDAFVIEPMTEKKTH

1861 KTEEAAITKKTLSLKKKMCASQRKQSCQEESLAVQDVNMEEDSFFMESMSFKKKPKTEES

1921 IPTHKLSS LKKKCT IYGK ICHFRKP PVLQTT ICGAMS S IKKPTTEKETLFQELSVLQEKH

1981 TTEHEMS ILKKSLALQKTNFKEDSLVKES LAFKKKPSTEEA IMMPVILKEQCMTEGKRSR

2041 LKPLVLQEITSGEKSLIMKPLS IKEKPSTEKESFSQEPSALQKKHTTQEEVS ILKEPSSL

2101 LKSPTEESPFDEALAFTKKCTIEEAPPTKKPLILKRKHATQGTMSHLKKPLILQTTSGEK

2161 SLIKEPLPFKEEKVSLKKKCTTQEMMS ICPELLDFQDMIGEDKNSFFMEPMSFRKNPTTE

2221 ETVLTKTSLSLQEKKITQGKMSHLKKPLVLQKITSEEESFYKKLLPFKMKSTTEEKFLSQ

2281 EPSALKEKHTTLQEVSLSKESLAIQEKATTEEEFSQELFSLHVKHTNKSGSLFQEALVLQ

2341 EKTDAEEDSLKNLLALQEKSTMEEESLINKLLALKEELSAEAATNIQTQLSLKKKSTS HG

2401 KVFFLKKQLALNETINEEEFLNKQPLALEGYPSIAEGETLFKKLLAMQEEPS IEKEAVLK

2461 EPTIDTEAHFKEPLALQEEPSTEKEAVLKEPSVDTEAHFKETLALQEKPS IEQEALFKRH

2521 SALWEKPSTEKETIFKESLDLQEKPSIKKETLLKKPLALKMSTINEAVLFEDMIALNEKP

2581 TTGKELSFKEPLALQESPTYKEDTFLKTLLVPQVGTSPNVSSTAPESITSKSSIATMTSV

2641 GKSGTINEAFLFEDMITLNEKPTTGKELSFKEPLALQESPTCKEDTFLETFLIPQIGTSP

2701 YVFSTTPESITEKSSIATMTSVGKSRTTTESSACESASDKPVSPQAKGTPKEITPREDID

2761 EDSSDPSFNPMYAKEIFSYMKEREEQFILTDYMNRQIEITSDMRAILVDWLVEVQVSFEM

2821 THETLYLAVKLVDLYLMKAVCKKDKLQLLGATAFMIAAKFEEHNSPRVDDFVYICDDNYQ

2881 RSEVLSMEINILNVLKCDINIPIAYHELRRYARCIHTNMKTLTLSRYICEMTLQEYHYVQ

2941 EKASKLAAASLLLALYMKKLGYWVPFLEHYSGYSISELHPLVRQLNKLLTFSSYDSLKAV

3001 YYKYSHPVFFEVAKIPALDMLKLEEILNCDCEAQGLVL *

Color legend (SMART domains):

red: Ankyrin repeats

green: Cyclin-like

The first nucleotide/peptide of CCNB3 is indicated in blue 
Supplementary Figure 6. Impact of BCOR-CCNB3 or DeltaCCNB3 on cell cycle phases. Mean $+/$ - standard deviation of triplicates is shown.

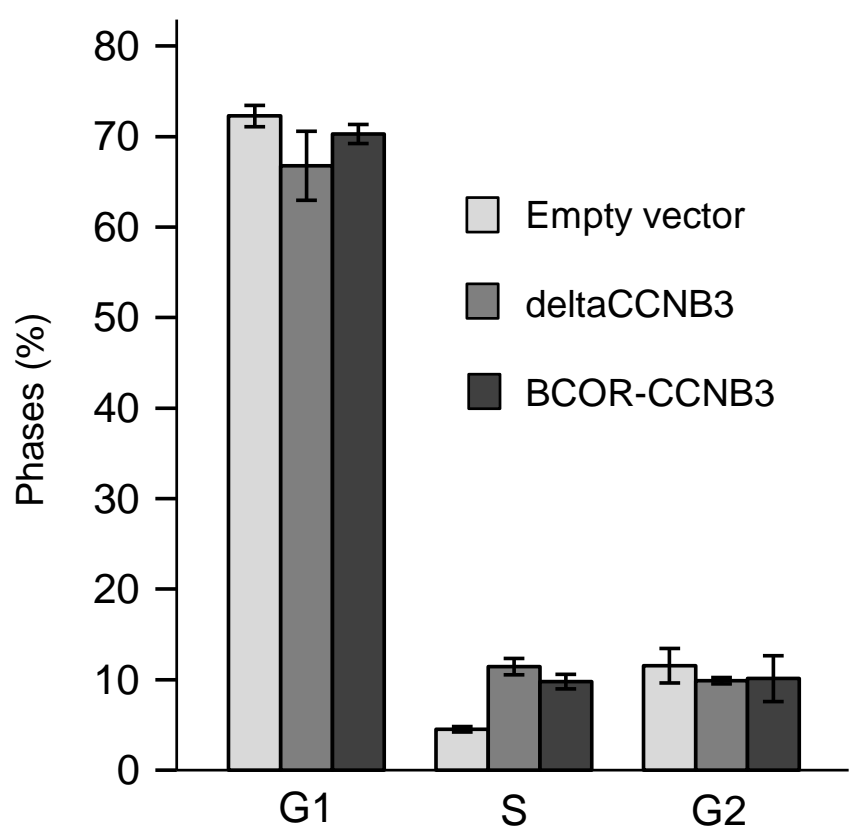

